\title{
Synthesis of 1,2-Benzothiazines by a Rhodium-Catalyzed Domino C-H Activation/Cyclization/Elimination Process from $S$-Aryl Sulfoximines and Pyridotriazoles
}

\author{
Woo Hyung Jeon, ${ }^{\dagger}$ Jeong-Yu Son, ${ }^{\dagger}$ Ji Eun Kim, and Phil Ho Lee* \\ National Creative Research Initiative Center for Catalytic Organic Reactions, Department of Chemistry, \\ Kangwon National University, Chuncheon 24341, Republic of Korea \\ FAX: (+)-82-33-259-5667. E-mail: phlee@kangwon.ac.kr
}

Contents

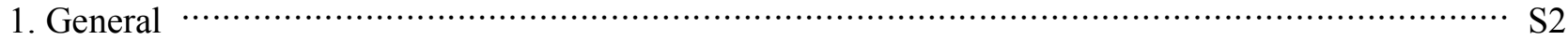

2. Preparation of Substrates

3. General procedure for Rh-catalyzed reaction of sulfoximines and pyridotriazoles …................. S4

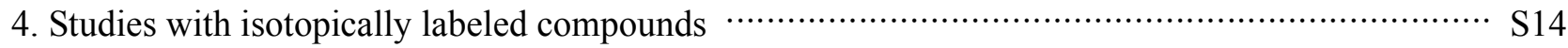

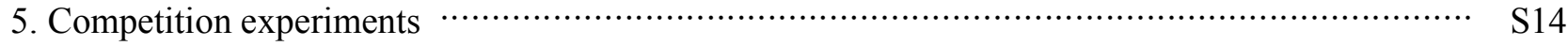

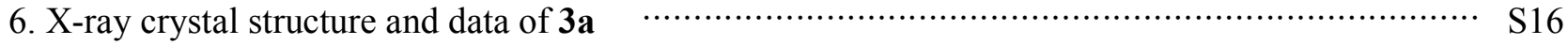

7. References

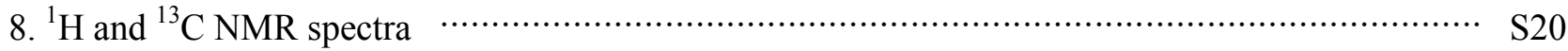




\section{Experimental Section}

1. General Commercial available reagents were used without purification. All reaction mixtures were stirred magnetically and were monitored by thin-layer chromatography using silica gel pre-coated glass plates, which were visualized with UV light and then, developed using either iodine or a solution of anisaldehyde. Flash column chromatography was carried out using silica gel (230-400 mesh). ${ }^{1} \mathrm{H}$ NMR (400 MHz) and ${ }^{13} \mathrm{C}$ NMR (100 MHz) spectra were recorded on NMR spectrometer. Deuterated chloroform was used as the solvent, and chemical shift values $(\delta)$ are reported in parts per million relative to the residual signals of this solvent $\left(\delta 7.26\right.$ for ${ }^{1} \mathrm{H}$ and $\delta 77.16$ for $\left.{ }^{13} \mathrm{C}\right)$. Infrared spectra were recorded on FT-IR spectrometer as either a thin film pressed between two sodium chloride plates or as a solid suspended in a potassium bromide disk. High resolution mass spectra (HRMS) were obtained by fast atom bombardment (FAB) using a double focusing magnetic sector mass spectrometer and electron impact (EI) ionization technique (magnetic sector-electric sector double focusing mass analyzer) from the KBSI (Korea Basic Science Institute). Melting points were determined in open capillary tube using Electrothermal 9100 apparatus. The rhodium complex $\left[\mathrm{Cp}^{*} \mathrm{Rh}(\mathrm{MeCN})_{3}\right]\left[\mathrm{SbF}_{6}\right]_{2}{ }^{1}$ was prepared according to the literature protocols. 


\section{Preparation of Substrates}

Sulfoximines $(\mathbf{1})^{2}$, pyridotriazoles $(\mathbf{2})^{3}$ and diazo compounds ${ }^{4}$ were prepared by reported method.

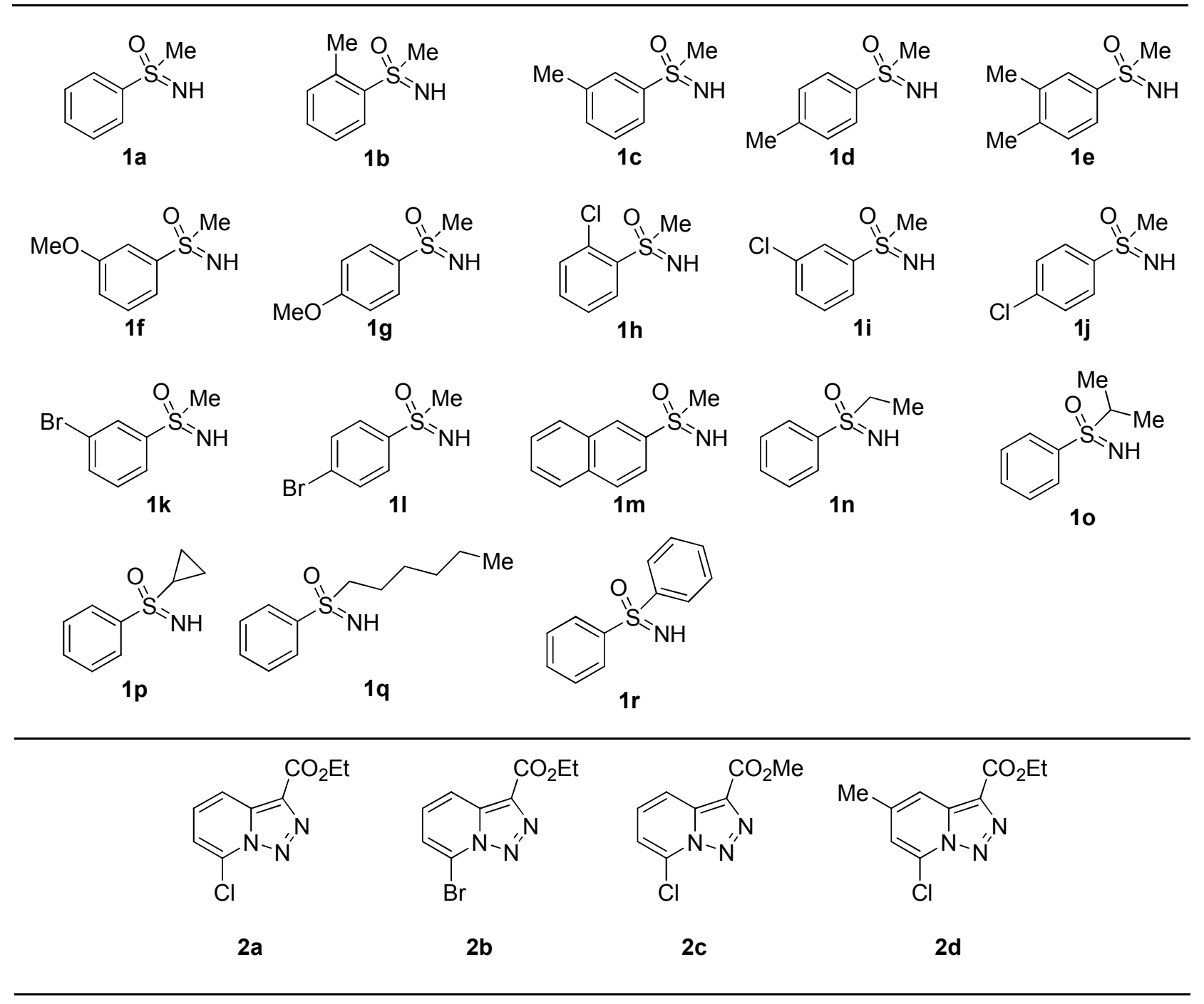<smiles>CCOC(=O)C(=N)C(C)=O</smiles> 


\section{General procedure for Rh-catalyzed reaction of sulfoximines and pyridotriazoles}

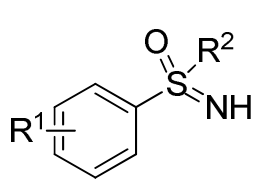

1<smiles>[R]Oc1nnn2cc[Z3]([R])cc12</smiles>

2
$\left[\mathrm{Cp}{ }^{*} \mathrm{Rh}(\mathrm{MeCN})_{3}\right]\left(\mathrm{SbF}_{6}\right)_{2}(3 \mathrm{~mol} \%)$

$\mathrm{NaOAc}(1.0$ equiv), toluene $100^{\circ} \mathrm{C}, 2 \mathrm{~h}$

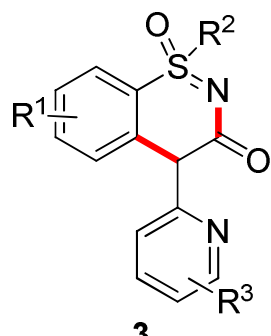

3

To a screw-top V-vial, sulfoximine (1) (1.2 equiv, $0.24 \mathrm{mmol}$ ), pyridotriazole (2) (1.0 equiv, 0.2 $\mathrm{mmol}),\left[\mathrm{Cp}^{*} \mathrm{Rh}(\mathrm{MeCN})_{3}\right]\left[\mathrm{SbF}_{6}\right]_{2}(3 \mathrm{~mol} \%)$, and $\mathrm{NaOAc}(1.0$ equiv, $0.2 \mathrm{mmol})$ in toluene $(2.0 \mathrm{~mL})$ were added. The resulting mixture was stirred at $100{ }^{\circ} \mathrm{C}$ for $2 \mathrm{~h}$ under argon. After celite filtration and evaporation of the solvents in vacuo, the crude product was purified by column chromatography on silica gel using EtOAc: $\mathrm{CH}_{2} \mathrm{Cl}_{2}=1: 1$.

4-(6-Chloropyridin-2-yl)-1-methylbenzo[e][1,2]thiazin-3(4H)-one 1-oxide (3a): Yield : $50.8 \mathrm{mg}$ $(83 \%) ; R_{f}=0.3$ (EtOAc: $\mathrm{CH}_{2} \mathrm{Cl}_{2}=1: 1$ ); White Solid; Melting point: $184-186{ }^{\circ} \mathrm{C} ;{ }^{1} \mathrm{H} \mathrm{NMR}(400 \mathrm{MHz}$, $\left.\mathrm{CDCl}_{3}\right) \delta 8.10(\mathrm{dd}, J=7.7,1.4 \mathrm{~Hz}, 1 \mathrm{H}), 7.71-7.65(\mathrm{~m}, 2 \mathrm{H}), 7.58(\mathrm{td}, J=7.6,1.3 \mathrm{~Hz}, 1 \mathrm{H}), 7.54(\mathrm{dd}, J=$ 7.5, 0.6 Hz, 1H), 7.27-7.25 (m, 1H), 7.19 (dd, $J=7.9,0.7 \mathrm{~Hz}, 1 \mathrm{H}), 5.05(\mathrm{~s}, 1 \mathrm{H}), 3.83(\mathrm{~s}, 3 \mathrm{H}) ;{ }^{13} \mathrm{C}$ NMR $\left(100 \mathrm{MHz}, \mathrm{CDCl}_{3}\right) \delta 168.9,159.0,151.2,140.5,138.1,135.3,129.8,129.3,129.2,126.4,123.6,122.7$, 54.1, 47.6; IR (film): 3075, 1645, 1325, 1159, 1042, $754 \mathrm{~cm}^{-1}$; HRMS (EI): $m / z$ calcd for $\mathrm{C}_{14} \mathrm{H}_{11} \mathrm{ClN}_{2} \mathrm{O}_{2} \mathrm{~S}$ : 306.0230; found: 306.0231

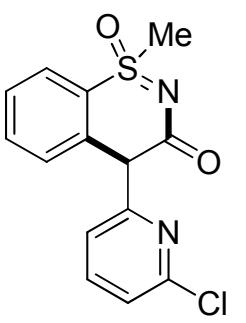

$3 a$

4-(6-Chloropyridin-2-yl)-1,8-dimethylbenzo[e][1,2]thiazin-3(4H)-one 1-oxide (3b): Yield : $63.4 \mathrm{mg}$ (99\%); $R_{f}=0.3$ (EtOAc: $\mathrm{CH}_{2} \mathrm{Cl}_{2}=1: 1$ ); Ivory solid; Melting point: $178-180{ }^{\circ} \mathrm{C} ;{ }^{1} \mathrm{H} \mathrm{NMR}(400 \mathrm{MHz}$, $\left.\mathrm{CDCl}_{3}\right) \delta 7.67(\mathrm{t}, J=7.8 \mathrm{~Hz}, 1 \mathrm{H}), 7.51-7.47(\mathrm{~m}, 2 \mathrm{H}), 7.35(\mathrm{~d}, J=7.6 \mathrm{~Hz}, 1 \mathrm{H}), 7.17(\mathrm{dd}, J=8.6,0.6 \mathrm{~Hz}$, $1 \mathrm{H}), 7.08(\mathrm{~d}, J=7.7 \mathrm{~Hz}, 1 \mathrm{H}), 5.01(\mathrm{~s}, 1 \mathrm{H}), 3.87(\mathrm{~s}, 3 \mathrm{H}), 2.85(\mathrm{~s}, 3 \mathrm{H}) ;{ }^{13} \mathrm{C} \mathrm{NMR}\left(100 \mathrm{MHz}, \mathrm{CDCl}_{3}\right) \delta$ $168.3,159.3,151.2,140.5,138.9,138.6,134.5,132.6,127.9,127.7,123.5,122.5,54.8,46.5,21.0$; IR (film): 3060, 1645, 1331, 1311, 1047, $770 \mathrm{~cm}^{-1}$; HRMS (EI): $\mathrm{m} / z$ calcd for $\mathrm{C}_{15} \mathrm{H}_{13} \mathrm{ClN}_{2} \mathrm{O}_{2} \mathrm{~S}: 320.0386$; found: 320.0390 


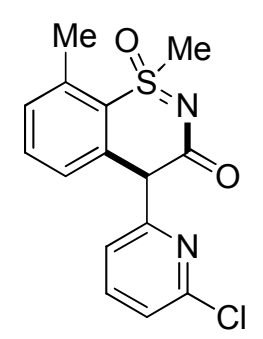

3b

4-(6-Chloropyridin-2-yl)-1,8-dimethylbenzo[e][1,2]thiazin-3(4H)-one 1-oxide (3c): Yield : $55.0 \mathrm{mg}$ $(86 \%) ; R_{f}=0.3$ (EtOAc: $\mathrm{CH}_{2} \mathrm{Cl}_{2}=1: 1$ ); Yellow solid; Melting point: $208-210{ }^{\circ} \mathrm{C} ;{ }^{1} \mathrm{H} \mathrm{NMR}(400 \mathrm{MHz}$, $\left.\mathrm{CDCl}_{3}\right) \delta 7.88(\mathrm{~s}, 1 \mathrm{H}), 7.68(\mathrm{t}, J=7.8 \mathrm{~Hz}, 1 \mathrm{H}), 7.52(\mathrm{dd}, J=7.5,0.6 \mathrm{~Hz}, 1 \mathrm{H}), 7.46(\mathrm{dd}, J=7.9,1.1 \mathrm{~Hz}$ $1 \mathrm{H}), 7.18(\mathrm{dd}, J=8.0,0.7 \mathrm{~Hz}, 1 \mathrm{H}), 7.14(\mathrm{~d}, J=8.0 \mathrm{~Hz}, 1 \mathrm{H}), 4.99(\mathrm{~s}, 1 \mathrm{H}), 3.82(\mathrm{~s}, 3 \mathrm{H}), 2.49(\mathrm{~s}, 3 \mathrm{H}) ;{ }^{13} \mathrm{C}$ NMR $\left(100 \mathrm{MHz}, \mathrm{CDCl}_{3}\right) \delta 169.2,159.2,151.2,140.4,139.9,136.3,135.1,129.6,128.8,126.4,123.5$, 122.6, 53.9, 47.6, 21.3; IR (film): 3054, 1650, 1308, 1234, 1045, $770 \mathrm{~cm}^{-1}$; HRMS (EI): $\mathrm{m} / z$ calcd for $\mathrm{C}_{15} \mathrm{H}_{13} \mathrm{ClN}_{2} \mathrm{O}_{2} \mathrm{~S}$ : 320.0386; found: 320.0390<smiles>Cc1ccc2c(c1)S(C)(=O)=NC(=O)C2c1cccc(Cl)n1</smiles>

$3 c$

4-(6-Chloropyridin-2-yl)-1,6-dimethylbenzo[e][1,2]thiazin-3(4H)-one 1-oxide (3d): Yield : $51.2 \mathrm{mg}$ $(80 \%) ; R_{f}=0.3$ (EtOAc: $\mathrm{CH}_{2} \mathrm{Cl}_{2}=1: 1$ ); Ivory solid; Melting point: $215-217{ }^{\circ} \mathrm{C} ;{ }^{1} \mathrm{H} \mathrm{NMR}(400 \mathrm{MHz}$, $\left.\mathrm{CDCl}_{3}\right) \delta 7.96(\mathrm{~d}, J=8.1 \mathrm{~Hz}, 1 \mathrm{H}), 7.69(\mathrm{t}, J=7.7 \mathrm{~Hz}, 1 \mathrm{H}), 7.53(\mathrm{~d}, J=7.5 \mathrm{~Hz}, 1 \mathrm{H}), 7.40(\mathrm{~d}, J=8.0 \mathrm{~Hz}$, $1 \mathrm{H}), 7.19(\mathrm{~d}, J=8.0 \mathrm{~Hz}, 1 \mathrm{H}), 7.03(\mathrm{~s}, 1 \mathrm{H}), 4.97(\mathrm{~s}, 1 \mathrm{H}), 3.80(\mathrm{~s}, 3 \mathrm{H}), 2.40(\mathrm{~s}, 3 \mathrm{H}) ;{ }^{13} \mathrm{C} \mathrm{NMR}(100 \mathrm{MHz}$, $\left.\mathrm{CDCl}_{3}\right) \delta 169.2,159.2,151.2,146.7,140.4,138.1,130.2,130.1,126.4,126.3,123.5,122.6,54.2,47.7$, 21.9; IR (film): 3055, 1737, 1324, 1307, 1045, $772 \mathrm{~cm}^{-1}$; HRMS (EI): $m / z$ calcd for $\mathrm{C}_{15} \mathrm{H}_{13} \mathrm{ClN}_{2} \mathrm{O}_{2} \mathrm{~S}$ : 320.0386; found: 320.0387

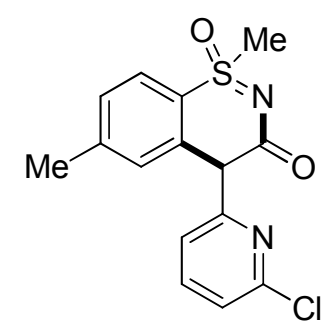

3d 
4-(6-Chloropyridin-2-yl)-1,6,7-trimethylbenzo[e][1,2]thiazin-3(4H)-one 1-oxide (3e): Yield : $53.4 \mathrm{mg}$ $(80 \%) ; R_{f}=0.4$ (EtOAc: $\mathrm{CH}_{2} \mathrm{Cl}_{2}=1: 1$ ); Ivory solid; Melting point: $248-250{ }^{\circ} \mathrm{C} ;{ }^{1} \mathrm{H} \mathrm{NMR}(400 \mathrm{MHz}$, $\left.\mathrm{CDCl}_{3}\right) \delta 7.81(\mathrm{~s}, 1 \mathrm{H}), 7.68(\mathrm{t}, J=7.7 \mathrm{~Hz}, 1 \mathrm{H}), 7.51(\mathrm{~d}, J=7.5 \mathrm{~Hz}, 1 \mathrm{H}), 7.17(\mathrm{~d}, J=8.0 \mathrm{~Hz}, 1 \mathrm{H}), 6.98(\mathrm{~s}$, $1 \mathrm{H}), 4.94(\mathrm{~s}, 1 \mathrm{H}), 3.80(\mathrm{~s}, 3 \mathrm{H}), 2.38(\mathrm{~s}, 3 \mathrm{H}), 2.30(\mathrm{~s}, 3 \mathrm{H}) ;{ }^{13} \mathrm{C} \mathrm{NMR}\left(100 \mathrm{MHz}, \mathrm{CDCl}_{3}\right) \delta 169.5,159.4$, $151.2,145.6,140.4,138.7,135.5,130.4,126.7,126.1,123.4,122.6,53.8,47.7,20.4$, 19.8; IR (film): 3056, 1645, 1326, 1230, 1067, $772 \mathrm{~cm}^{-1}$; HRMS (EI): $\mathrm{m} / z$ calcd for $\mathrm{C}_{16} \mathrm{H}_{15} \mathrm{ClN}_{2} \mathrm{O}_{2} \mathrm{~S}: 334.0543$; found: 334.0544

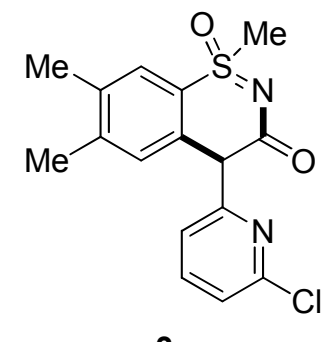

$3 e$

4-(6-Chloropyridin-2-yl)-7-methoxy-1-methylbenzo[e][1,2]thiazin-3(4H)-one 1-oxide (3f): Yield : $49.7 \mathrm{mg}(74 \%) ; R_{f}=0.4$ (EtOAc: $\mathrm{CH}_{2} \mathrm{Cl}_{2}=1: 1$ ); Ivory solid; Melting point: $208-210{ }^{\circ} \mathrm{C}$; ${ }^{1} \mathrm{H} \mathrm{NMR}(400$ $\left.\mathrm{MHz}, \mathrm{CDCl}_{3}\right) \delta 7.70(\mathrm{dd}, J=7.9,0.5 \mathrm{~Hz}, 1 \mathrm{H}), 7.66(\mathrm{t}, J=7.7 \mathrm{~Hz}, 1 \mathrm{H}), 7.60-7.55(\mathrm{~m}, 2 \mathrm{H}), 7.15(\mathrm{~d}, J=8.0$ $\mathrm{Hz}, 2 \mathrm{H}), 5.21(\mathrm{~s}, 1 \mathrm{H}), 3.85(\mathrm{~s}, 3 \mathrm{H}), 3.81(\mathrm{~s}, 3 \mathrm{H}) ;{ }^{13} \mathrm{C} \mathrm{NMR}\left(100 \mathrm{MHz}, \mathrm{CDCl}_{3}\right) \delta 169.4,158.6,156.2$, 150.6, 139.9, 129.9, 129.8, 126.8, 123.8, 123.1, 117.6, 116.2, 56.3, 48.8, 47.9; IR (film): 3099, 1737, 1325, 1306, 1083, $786 \mathrm{~cm}^{-1}$; HRMS (EI): $\mathrm{m} / z$ calcd for $\mathrm{C}_{15} \mathrm{H}_{13} \mathrm{ClN}_{2} \mathrm{O}_{3} \mathrm{~S}: 336.0335$; found: 336.0338

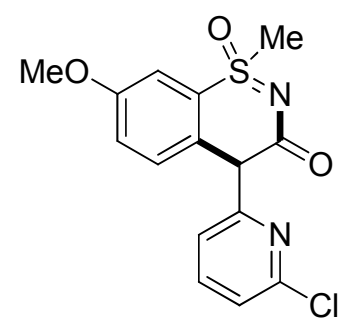

$3 \mathbf{f}$

4-(6-Chloropyridin-2-yl)-6-methoxy-1-methylbenzo[e][1,2]thiazin-3(4H)-one 1-oxide (3g): Yield : $48.4 \mathrm{mg}(72 \%) ; R_{f}=0.4$ (EtOAc: $\mathrm{CH}_{2} \mathrm{Cl}_{2}=1: 1$ ); Ivory solid; Melting point: $200-203{ }^{\circ} \mathrm{C}$; ${ }^{1} \mathrm{H}$ NMR $(400$ $\left.\mathrm{MHz}, \mathrm{CDCl}_{3}\right) \delta 8.00(\mathrm{~d}, J=8.8 \mathrm{~Hz}, 1 \mathrm{H}), 7.69(\mathrm{t}, J=7.8 \mathrm{~Hz}, 1 \mathrm{H}), 7.52(\mathrm{~d}, J=7.5 \mathrm{~Hz}, 1 \mathrm{H}), 7.19(\mathrm{~d}, J=$ $8.0 \mathrm{~Hz}, 1 \mathrm{H}), 7.08(\mathrm{~d}, J=8.8 \mathrm{~Hz}, 1 \mathrm{H}), 6.66(\mathrm{~s}, 1 \mathrm{H}), 4.96(\mathrm{~s}, 1 \mathrm{H}), 3.85(\mathrm{~s}, 3 \mathrm{H}), 3.78(\mathrm{~s}, 3 \mathrm{H}) ;{ }^{13} \mathrm{C} \mathrm{NMR}(100$ $\left.\mathrm{MHz}, \mathrm{CDCl}_{3}\right) \delta 169.2,164.6,159.1,151.2,140.5,140.4,128.6,123.6,122.6,120.7,115.4,114.1,56.0$, 54.6, 47.8; IR (film): 3069, 1738, 1327, 1306, 1043, $773 \mathrm{~cm}^{-1}$; HRMS (EI): $\mathrm{m} / z$ calcd for $\mathrm{C}_{15} \mathrm{H}_{13} \mathrm{ClN}_{2} \mathrm{O}_{3} \mathrm{~S}: 336.0335$; found : 336.0337 


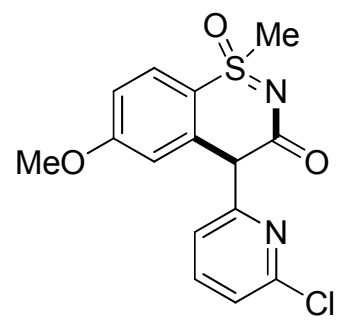

$3 \mathrm{~g}$

8-Chloro-4-(6-chloropyridin-2-yl)-1-methylbenzo[e][1,2]thiazin-3(4H)-one 1-oxide (3h): Yield : 54.4 mg $(80 \%) R_{f}=0.5$ (EtOAc: $\left.\mathrm{CH}_{2} \mathrm{Cl}_{2}=1: 1\right)$; Pale yellow solid; Melting point: $168-170{ }^{\circ} \mathrm{C}$; ${ }^{1} \mathrm{HNMR}(400$ $\left.\mathrm{MHz}, \mathrm{CDCl}_{3}\right) \delta 7.68(\mathrm{t}, J=7.8 \mathrm{~Hz}, 1 \mathrm{H}), 7.58-7.52(\mathrm{~m}, 2 \mathrm{H}), 7.49(\mathrm{dd}, J=7.5,0.6 \mathrm{~Hz}, 1 \mathrm{H}), 7.20-7.15(\mathrm{~m}$, 2H), $5.03(\mathrm{~s}, 1 \mathrm{H}), 3.97(\mathrm{~s}, 3 \mathrm{H}) ;{ }^{13} \mathrm{C} \mathrm{NMR}\left(100 \mathrm{MHz}, \mathrm{CDCl}_{3}\right) \delta 167.2,158.5,151.4,141.1,140.6,135.2$, 133.9, 131.8, 128.6, 127.6, 123.7, 122.3, 54.3, 45.5 ; IR (film): 3070, 1654, 1332, 1242, 1053, 777, 734 $\mathrm{cm}^{-1}$; HRMS (EI): $\mathrm{m} / z$ calcd for $\mathrm{C}_{14} \mathrm{H}_{10} \mathrm{Cl}_{2} \mathrm{~N}_{2} \mathrm{O}_{2} \mathrm{~S}$ : 339.9840; found: 339.9842<smiles>CS1(=O)=NC(=O)C(c2cccc(Cl)n2)c2cccc(Cl)c21</smiles>

$3 \mathrm{~h}$

7-Chloro-4-(6-chloropyridin-2-yl)-1-methylbenzo[e][1,2]thiazin-3(4H)-one 1-oxide (3i): Yield : 44.2 $\operatorname{mg}(65 \%) R_{f}=0.5$ (EtOAc: $\left.\mathrm{CH}_{2} \mathrm{Cl}_{2}=1: 1\right)$; White solid; Melting point: $248-250{ }^{\circ} \mathrm{C} ;{ }^{1} \mathrm{HNMR}(400 \mathrm{MHz}$, $\left.\mathrm{CDCl}_{3}\right) \delta 8.07(\mathrm{~d}, J=7.8 \mathrm{~Hz}, 1 \mathrm{H}), 7.72(\mathrm{t}, J=7.6 \mathrm{~Hz}, 2 \mathrm{H}), 7.67(\mathrm{~d}, J=6.8 \mathrm{~Hz}, 1 \mathrm{H}), 7.60(\mathrm{t}, J=7.9 \mathrm{~Hz}$, $1 \mathrm{H}), 7.21(\mathrm{dd}, J=7.6,0.6 \mathrm{~Hz}, 1 \mathrm{H}), 5.31(\mathrm{~s}, 1 \mathrm{H}), 3.86(\mathrm{~s}, 3 \mathrm{H}) ;{ }^{13} \mathrm{C} \mathrm{NMR}\left(100 \mathrm{MHz}, \mathrm{CDCl}_{3}\right) \delta 168.1$, 157.0, 150.8, 140.2, 136.1, 135.7, 134.9, 131.6, 130.0, 125.1, 124.5, 123.6, 52.2, 47.8 ; IR (film): 3072, 1654, 1325, 1235, 1045, 786, $733 \mathrm{~cm}^{-1}$; HRMS (EI): $\mathrm{m} / z$ calcd for $\mathrm{C}_{14} \mathrm{H}_{10} \mathrm{Cl}_{2} \mathrm{~N}_{2} \mathrm{O}_{2} \mathrm{~S}$ : 339.9840; found: 339.9844

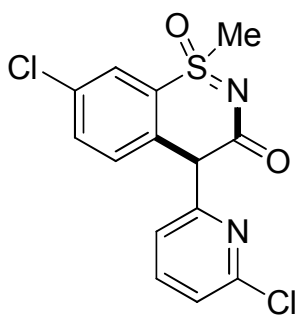

$3 \mathbf{i}$ 
6-Chloro-4-(6-chloropyridin-2-yl)-1-methylbenzo[e][1,2] thiazin-3(4H)-one 1-oxide (3j): Yield : 41.5 mg $(61 \%) R_{f}=0.5$ (EtOAc: $\left.\mathrm{CH}_{2} \mathrm{Cl}_{2}=1: 1\right)$; Yellow oil; ${ }^{1} \mathrm{H}$ NMR $\left(400 \mathrm{MHz}, \mathrm{CDCl}_{3}\right) \delta 8.04(\mathrm{~d}, J=8.4 \mathrm{~Hz}$, $1 \mathrm{H}), 7.72(\mathrm{t}, J=7.8 \mathrm{~Hz}, 1 \mathrm{H}), 7.59(\mathrm{dd}, J=8.4,1.9 \mathrm{~Hz}, 1 \mathrm{H}), 7.54(\mathrm{~d}, J=7.5 \mathrm{~Hz}, 1 \mathrm{H}), 7.25(\mathrm{~d}, J=1.9 \mathrm{~Hz}$, $1 \mathrm{H}), 7.22(\mathrm{~d}, J=8.0 \mathrm{~Hz}, 1 \mathrm{H}), 5.00(\mathrm{~s}, 1 \mathrm{H}), 3.82(\mathrm{~s}, 3 \mathrm{H}) ;{ }^{13} \mathrm{C} \mathrm{NMR}\left(100 \mathrm{MHz}, \mathrm{CDCl}_{3}\right) \delta 168.2,158.3$, $151.3,141.8,140.7,140.0,129.9,129.8,128.0,127.7,123.8,122.7,54.0,47.7$; IR (film): 3087, 1738, 1327, 1309, 1071, $791 \mathrm{~cm}^{-1}$; HRMS (EI): $\mathrm{m} / z$ calcd for $\mathrm{C}_{14} \mathrm{H}_{10} \mathrm{Cl}_{2} \mathrm{~N}_{2} \mathrm{O}_{2} \mathrm{~S}: 339.9840$; found: 339.9839<smiles>CS1(=O)=NC(=O)C(c2cccc(Cl)n2)c2cc(Cl)ccc21</smiles>

3j

7-Bromo-4-(6-chloropyridin-2-yl)-1-methylbenzo[e][1,2]thiazin-3(4H)-one 1-oxide (3k): Yield : 57.6 $\operatorname{mg}(75 \%) R_{f}=0.5$ (EtOAc: $\left.\mathrm{CH}_{2} \mathrm{Cl}_{2}=1: 1\right)$; White solid; Melting point: $235-237{ }^{\circ} \mathrm{C} ;{ }^{1} \mathrm{HNMR}(400 \mathrm{MHz}$, $\left.\mathrm{CDCl}_{3}\right) \delta 8.11(\mathrm{dd}, J=7.9,0.7 \mathrm{~Hz}, 1 \mathrm{H}), 7.91(\mathrm{dd}, J=8.0,1.2 \mathrm{~Hz}, 1 \mathrm{H}), 7.72(\mathrm{~d}, J=1.8 \mathrm{~Hz}, 1 \mathrm{H}), 7.71(\mathrm{~s}$, $1 \mathrm{H}), 7.53(\mathrm{t}, J=7.9 \mathrm{~Hz}, 1 \mathrm{H}), 7.23-7.18(\mathrm{~m}, 1 \mathrm{H}), 5.30(\mathrm{~s}, 1 \mathrm{H}), 3.86(\mathrm{~s}, 3 \mathrm{H}) ;{ }^{13} \mathrm{C} \mathrm{NMR}\left(100 \mathrm{MHz}, \mathrm{CDCl}_{3}\right)$ $\delta 168.2,156.9,150.8,140.2,139.5,137.0,131.9,130.4,125.8,125.2,124.9,123.5,54.7,47.7$; IR (film): $3069,1652,1322,1236,1044,780,619 \mathrm{~cm}^{-1}$; HRMS (EI): $\mathrm{m} / z$ calcd for $\mathrm{C}_{14} \mathrm{H}_{10} \mathrm{BrClN}_{2} \mathrm{O}_{2} \mathrm{~S}: 383.9335$; found: 383.9338<smiles>CS1(=O)=NC(=O)C(c2cccc(Cl)n2)c2ccc(Br)cc21</smiles>

3k

6-Bromo-4-(6-chloropyridin-2-yl)-1-methylbenzo[e][1,2]thiazin-3(4H)-one 1-oxide (3I): Yield : 57.6 $\operatorname{mg}(75 \%) R_{f}=0.5$ (EtOAc: $\left.\mathrm{CH}_{2} \mathrm{Cl}_{2}=1: 1\right)$; Ivory solid; Melting point: $153-156{ }^{\circ} \mathrm{C} ;{ }^{1} \mathrm{HNMR}(400 \mathrm{MHz}$, $\left.\mathrm{CDCl}_{3}\right) \delta 7.96(\mathrm{~d}, J=8.4 \mathrm{~Hz}, 1 \mathrm{H}), 7.76-7.70(\mathrm{~m}, 2 \mathrm{H}), 7.54(\mathrm{dd}, J=7.5,0.7 \mathrm{~Hz}, 1 \mathrm{H}), 7.42(\mathrm{~d}, J=1.8 \mathrm{~Hz}$, 1H), $7.22(\mathrm{~d}, J=8.0 \mathrm{~Hz}, 1 \mathrm{H}), 4.98(\mathrm{~s}, 1 \mathrm{H}), 3.82(\mathrm{~s}, 3 \mathrm{H}) ;{ }^{13} \mathrm{C} \mathrm{NMR}\left(100 \mathrm{MHz}, \mathrm{CDCl}_{3}\right) \delta 168.1,158.3$, $151.4,140.7,139.9,132.8,132.7,130.4,128.3,127.9,123.9,122.8,53.9,47.7$; IR (film): 3086, 1737, 1405, 1308, 1065, 778, $657 \mathrm{~cm}^{-1}$; HRMS (EI): $\mathrm{m} / z$ calcd for $\mathrm{C}_{14} \mathrm{H}_{10} \mathrm{BrClN}_{2} \mathrm{O}_{2} \mathrm{~S}$ : 383.9335; found: 383.9331 


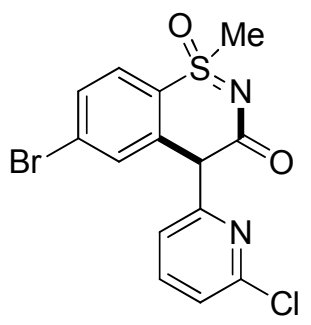

31

4-(6-Chloropyridin-2-yl)-1-methylnaphtho[2,3-e][1,2]thiazin-3(4H)-one 1-oxide (3m): Yield : 35.6 mg $(50 \%) R_{f}=0.4$ (EtOAc: $\mathrm{CH}_{2} \mathrm{Cl}_{2}=1: 1$ ); Ivory solid; Melting point: $260-263{ }^{\circ} \mathrm{C} ;{ }^{1} \mathrm{HNMR}(400 \mathrm{MHz}$, $\left.\mathrm{CDCl}_{3}\right) \delta 8.09(\mathrm{~s}, 2 \mathrm{H}), 7.98(\mathrm{~d}, J=9.5 \mathrm{~Hz}, 2 \mathrm{H}), 7.84(\mathrm{dd}, J=7.6,0.6 \mathrm{~Hz}, 1 \mathrm{H}), 7.73(\mathrm{t}, 7.8 \mathrm{~Hz}, 1 \mathrm{H}), 7.71-$ $7.67(\mathrm{~m}, 1 \mathrm{H}), 7.64-7.60(\mathrm{~m}, 1 \mathrm{H}), 7.15(\mathrm{dd}, J=7.9,0.7 \mathrm{~Hz}, 1 \mathrm{H}), 5.73(\mathrm{~s}, 1 \mathrm{H}), 3.08(\mathrm{~s}, 3 \mathrm{H}) ;{ }^{13} \mathrm{C} \mathrm{NMR}(100$ $\left.\mathrm{MHz}, \mathrm{CDCl}_{3}\right) \delta 169.0,158.5,151.1,140.5,136.2,136.0,130.4,129.7,129.64,129.61,128.9,126.4$, 124.3, 123.6, 123.3, 120.2, 51.5, 47.3; IR (film): 3065, 1645, 1369, 1332, 1075, $785 \mathrm{~cm}^{-1}$; HRMS (EI): $m / z$ calcd for $\mathrm{C}_{18} \mathrm{H}_{13} \mathrm{ClN}_{2} \mathrm{O}_{2} \mathrm{~S}: 356.0386$; found: 356.0387

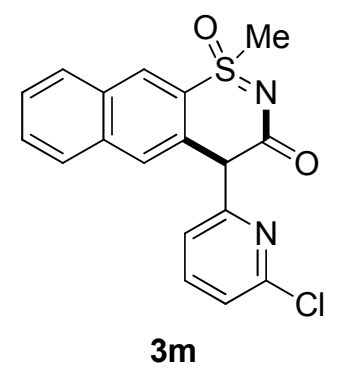

4-(6-Chloropyridin-2-yl)-1-ethylbenzo[e][1,2]thiazin-3(4H)-one 1-oxide (3n): Yield : $52.6 \mathrm{mg}(82 \%)$; $R_{f}=0.3$ (EtOAc: $\mathrm{CH}_{2} \mathrm{Cl}_{2}=1: 1$ ); Yellow oil; dr (1:5); ${ }^{1} \mathrm{HNMR}\left(400 \mathrm{MHz}, \mathrm{CDCl}_{3}\right) \delta 8.02$ (dd, $J=7.8,1.3$ $\mathrm{Hz}, 1 \mathrm{H}), 7.90(\mathrm{dd}, J=7.8,1.3 \mathrm{~Hz}, 0.2 \mathrm{H}), 7.71-7.65(\mathrm{~m}, 2.4 \mathrm{H}), 7.62-7.58(\mathrm{~m}, 1.4 \mathrm{H}), 7.55(\mathrm{dd}, J=7.5,0.6$ $\mathrm{Hz}, 1 \mathrm{H}), 7.36(\mathrm{~d}, J=7.7 \mathrm{~Hz}, 0.2 \mathrm{H}), 7.30-7.24(\mathrm{~m}, 1.2 \mathrm{H}), 7.19(\mathrm{dd}, J=8.0,0.7 \mathrm{~Hz}, 1 \mathrm{H}), 5.16(\mathrm{~s}, 0.2 \mathrm{H})$, $5.05(\mathrm{~s}, 1 \mathrm{H}), 4.09-3.95(\mathrm{~m}, 2 \mathrm{H}), 3.63-3.52(\mathrm{~m}, 0.4 \mathrm{H}), 1.59(\mathrm{t}, J=7.4 \mathrm{~Hz}, 3 \mathrm{H}), 1.31(\mathrm{t}, J=7.3 \mathrm{~Hz}, 0.6 \mathrm{H})$; ${ }^{13} \mathrm{C} \mathrm{NMR}\left(100 \mathrm{MHz}, \mathrm{CDCl}_{3}\right) \delta 171.2,169.3,159.0,151.1,140.4,140.0,139.5,138.5,135.2,135.0,130.8$, 130.0, 129.1, 129.0, 128.0, 127.2, 126.1, 125.2, 123.5, 122.8, 121.8, 54.5, 54.1, 53.7, 51.1, 7.7, 7.0; IR (film): 3073, 1647, 1323, 1267, 1078, $761 \mathrm{~cm}^{-1}$; HRMS (EI): $\mathrm{m} / z$ calcd for $\mathrm{C}_{15} \mathrm{H}_{13} \mathrm{ClN}_{2} \mathrm{O}_{2} \mathrm{~S}: 320.0386$; found: 320.0384 


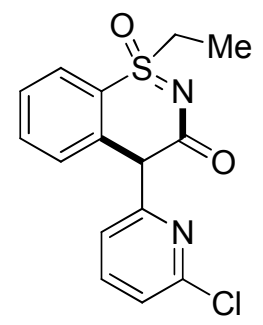

$3 n$

4-(6-Chloropyridin-2-yl)-1-isopropylbenzo[e][1,2]thiazin-3(4H)-one 1-oxide (3o): Yield : $40.8 \mathrm{mg}$ $(61 \%) ; R_{f}=0.3$ (EtOAc: $\left.\mathrm{CH}_{2} \mathrm{Cl}_{2}=1: 1\right)$; Ivory solid; Melting point: $82-84{ }^{\circ} \mathrm{C}$; $\mathrm{dr}(1: 1.7)$; ${ }^{1} \mathrm{H}$ NMR (400 $\left.\mathrm{MHz}, \mathrm{CDCl}_{3}\right) \delta 7.99(\mathrm{dd}, J=7.8,0.9 \mathrm{~Hz}, 1 \mathrm{H}), 7.91(\mathrm{dd}, J=7.8,1.1 \mathrm{~Hz}, 0.6 \mathrm{H}), 7.71-7.56(\mathrm{~m}, 6.7 \mathrm{H})$, 7.28-7.23 (m, 2.2H), 7.19 (d, $J=8.0 \mathrm{~Hz}, 1 \mathrm{H}), 5.13(\mathrm{~s}, 0.6 \mathrm{H}), 5.05(\mathrm{~s}, 1 \mathrm{H}), 4.46-4.40(\mathrm{~s}, 1 \mathrm{H}), 3.79-3.72$ $(\mathrm{m}, 0.6 \mathrm{H}), 1.59-1.54(\mathrm{~m}, 7.8 \mathrm{H}), 1.26(\mathrm{~d}, J=6.7 \mathrm{~Hz}, 1.8 \mathrm{H}) ;{ }^{13} \mathrm{C} \mathrm{NMR}\left(100 \mathrm{MHz}, \mathrm{CDCl}_{3}\right) \delta 171.1,169.6$, $159.0,158.9,151.1,151.0,140.3,140.0,139.8,139.0,135.1,135.0,130.6,130.2,128.9,128.6,128.0$, 127.2, 125.6, 125.3, 123.4, 123.1, 122.1, 60.1, 57.2, 54.0, 53.6, 16.5, 16.3, 14.4, 13.9; IR (film): 3061, 1650, 1405, 1320, 1075, $800 \mathrm{~cm}^{-1}$; HRMS (EI): $\mathrm{m} / z$ calcd for $\mathrm{C}_{16} \mathrm{H}_{15} \mathrm{ClN}_{2} \mathrm{O}_{2} \mathrm{~S}: 334.0543$; found: 334.0541

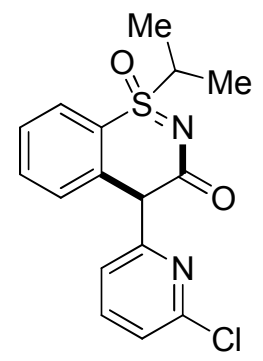

30

4-(6-Chloropyridin-2-yl)-1-cyclopropylbenzo[e][1,2]thiazin-3(4H)-one 1-oxide (3p): Yield : $40.0 \mathrm{mg}$ $(60 \%) ; R_{f}=0.3$ (EtOAc: $\left.\mathrm{CH}_{2} \mathrm{Cl}_{2}=1: 1\right)$; Yellow oil; dr (1:5); ${ }^{1} \mathrm{H} \mathrm{NMR}\left(400 \mathrm{MHz}, \mathrm{CDCl}_{3}\right) \delta 8.00(\mathrm{dd}, J=$ 7.8, 1.2 Hz, 0.2H), 7.95 (dd, $J=7.8,1.3 \mathrm{~Hz}, 1 \mathrm{H}), 7.69$ (t, $J=7.8 \mathrm{~Hz}, 1 \mathrm{H}), 7.63$ (td, $J=11.3,1.3 \mathrm{~Hz}$, 1.2H), 7.60-7.54 (m, 2.4H), $7.38(\mathrm{~d}, J=7.8 \mathrm{~Hz}, 0.2 \mathrm{H}), 7.32(\mathrm{~d}, J=2.7 \mathrm{~Hz}, 0.2 \mathrm{H}), 7.24(\mathrm{~d}, J=7.6 \mathrm{~Hz}$, $1.1 \mathrm{H}), 7.18(\mathrm{dd}, J=8.0,0.6 \mathrm{~Hz}, 1.1 \mathrm{H}), 5.18(\mathrm{~s}, 0.2 \mathrm{H}), 5.06(\mathrm{~s}, 1 \mathrm{H}), 3.83-3.77(\mathrm{~s}, 1 \mathrm{H}), 2.80-2.73(\mathrm{~m}$, $0.2 \mathrm{H}), 1.77-1.70(\mathrm{~m}, 1 \mathrm{H}), 1.55-1.50(\mathrm{~m}, 1 \mathrm{H}), 1.45-1.37(\mathrm{~m}, 0.4 \mathrm{H}), 1.30-1.21(\mathrm{~m}, 2.8 \mathrm{H}) ;{ }^{13} \mathrm{C}$ NMR $(100$ $\left.\mathrm{MHz}, \mathrm{CDCl}_{3}\right) \delta 171.8,169.7,159.0,158.4,151.1,140.4,139.9,138.6,138.2,134.9,134.5,130.6,129.9$, 129.7, 129.6, 129.1, 129.0, 126.6, 125.0, 123.5, 123.4, 122.8, 121.6, 54.2, 53.9, 35.3, 33.0, 7.3, 7.1, 5.6, 5.2; IR (film): 3056, 1654, 1316, 1268, 1076, $798 \mathrm{~cm}^{-1}$; HRMS (EI): $m / z$ calcd for $\mathrm{C}_{16} \mathrm{H}_{13} \mathrm{ClN}_{2} \mathrm{O}_{2} \mathrm{~S}$ : 332.0386; found: 332.0388 


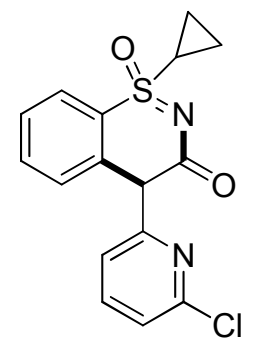

$3 p$

4-(6-Chloropyridin-2-yl)-1-hexylbenzo[e $][1,2]$ thiazin-3(4H)-one 1-oxide (3q): Yield : 56.5 mg (75 \%); $R_{f}=0.3$ (EtOAc: $\mathrm{CH}_{2} \mathrm{Cl}_{2}=1: 1$ ); Ivory solid; Melting point: $130-132{ }^{\circ} \mathrm{C}$; $\mathrm{dr}(1: 4) ;{ }^{1} \mathrm{H} \mathrm{NMR}(400 \mathrm{MHz}$, $\left.\mathrm{CDCl}_{3}\right) \delta 8.02(\mathrm{dd}, J=7.8,1.0 \mathrm{~Hz}, 1 \mathrm{H}), 7.90(\mathrm{dd}, J=7.8,1.0 \mathrm{~Hz}, 0.25 \mathrm{H}), 7.71-7.55(\mathrm{~m}, 5 \mathrm{H}), 7.34(\mathrm{~d}, J=$ $7.8 \mathrm{~Hz}, 0.25 \mathrm{H}), 7.29-7.22(\mathrm{~m}, 1.25 \mathrm{H}), 7.19(\mathrm{~d}, J=8.0 \mathrm{~Hz}, 1 \mathrm{H}), 5.15(\mathrm{~s}, 0.25 \mathrm{H}), 5.04(\mathrm{~s}, 1 \mathrm{H}), 3.97$ (t, $J=$ $8.1 \mathrm{~Hz}, 2 \mathrm{H}), 3.50$ (t, $J=8.1 \mathrm{~Hz}, 0.5 \mathrm{H}), 2.17-1.78(\mathrm{~m}, 2.5 \mathrm{H}), 1.55-1.46(\mathrm{~m}, 2.5 \mathrm{H}), 1.34-1.24(\mathrm{~m}, 6 \mathrm{H}), 0.9-$ $0.86(\mathrm{~m}, 4 \mathrm{H}) ;{ }^{13} \mathrm{C}$ NMR $\left(100 \mathrm{MHz}, \mathrm{CDCl}_{3}\right) \delta 169.2,159.1,158.6,151.1,140.5,140.4,140.0,139.3$, 138.3 , 135.2, 134.9, 130.7, 130.0, 129.0, 128.9, 128.5, 127.1, 126.8, 125.1, 123.5, 123.4, 122.9, 121.8, 59.9, 56.5, 54.1, 53.7, 31.4, 31.2, 28.0, 27.7, 22.8, 22.5, 22.4, 22.1, 14.1, 14.0; IR (film): 2927, 1646, 1431, 1321, 1228, 1077, $800 \mathrm{~cm}^{-1}$; HRMS (EI): $\mathrm{m} / z$ calcd for $\mathrm{C}_{19} \mathrm{H}_{21} \mathrm{ClN}_{2} \mathrm{O}_{2} \mathrm{~S}: 376.1012$; found: 376.1013

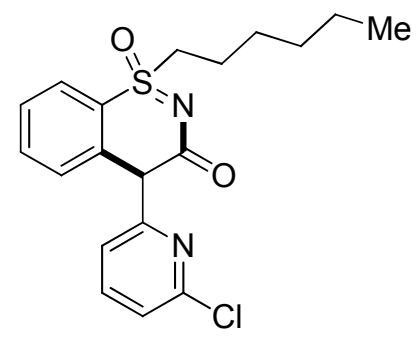

$3 q$

4-(6-Chloropyridin-2-yl)-1-phenylbenzo[e][1,2]thiazin-3(4H)-one 1-oxide (3r): Yield : $44.2 \mathrm{mg}$ $(60 \%) ; R_{f}=0.4$ (EtOAc: $\left.\mathrm{CH}_{2} \mathrm{Cl}_{2}=1: 1\right)$; Ivory solid; Melting point: $161-163{ }^{\circ} \mathrm{C}$; $\mathrm{dr}(1: 4)$; ${ }^{1} \mathrm{H} \mathrm{NMR}(400$ $\left.\mathrm{MHz}, \mathrm{CDCl}_{3}\right) \delta 8.46-8.43(\mathrm{~m}, 2 \mathrm{H}), 8.04-8.01(\mathrm{~m}, 0.5 \mathrm{H}), 7.77-7.74(\mathrm{~m}, 1 \mathrm{H}), 7.73(\mathrm{t}, J=7.8 \mathrm{~Hz}, 1 \mathrm{H}), 7.67-$ $7.65(\mathrm{~m}, 1.25 \mathrm{H}), 7.63-7.59(\mathrm{~m}, 3.5 \mathrm{H}), 7.57-7.50(\mathrm{~m}, 1.5 \mathrm{H}), 7.44(\mathrm{t}, J=8.0 \mathrm{~Hz}, 1.5 \mathrm{H}), 7.40-7.37(\mathrm{~m}, 0.5 \mathrm{H})$, 7.26-7.23 (m, 1.25H), $7.14(\mathrm{~d}, J=7.9 \mathrm{~Hz}, 1 \mathrm{H}), 5.29(\mathrm{~s}, 0.25 \mathrm{H}), 5.12(\mathrm{~s}, 1 \mathrm{H}) ;{ }^{13} \mathrm{C} \mathrm{NMR}(100 \mathrm{MHz}$, $\left.\mathrm{CDCl}_{3}\right) \delta 170.0,159.4,158.3,151.3,151.2,140.0,139.9,138.9,138.3,138.2,137.5,134.7,134.5,134.2$, $130.5,130.4,130.3,129.9,129.8,129.5,129.0,128.9,128.6,127.0,126.2,123.6,123.5,123.4,121.7$, 54.4, 51.7; IR (film): 3061, 2914, 1736, 1304, 1267, $797 \mathrm{~cm}^{-1}$; HRMS (EI): $\mathrm{m} / z$ calcd for $\mathrm{C}_{19} \mathrm{H}_{13} \mathrm{ClN}_{2} \mathrm{O}_{2} \mathrm{~S}: 368.0386$; found: 368.0389 


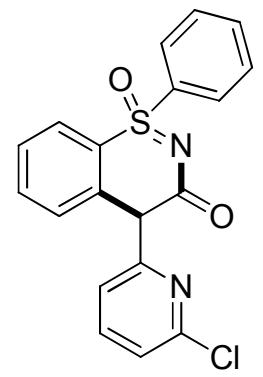

$3 r$

4-(6-Bromopyridin-2-yl)-1-methylbenzo[e $][1,2]$ thiazin-3(4H)-one 1-oxide (4a): Yield : $52.5 \mathrm{mg}$ $(75 \%) R_{f}=0.3$ (EtOAc: $\mathrm{CH}_{2} \mathrm{Cl}_{2}=1: 1$ ); White solid; Melting point: $149-151{ }^{\circ} \mathrm{C} ;{ }^{1} \mathrm{H} \mathrm{NMR}(400 \mathrm{MHz}$, $\left.\mathrm{CDCl}_{3}\right) \delta 8.10(\mathrm{dd}, J=7.7,1.4 \mathrm{~Hz}, 1 \mathrm{H}), 7.66(\mathrm{dd}, J=7.6,1.5 \mathrm{~Hz}, 1 \mathrm{H}), 7.63(\mathrm{dd}, J=7.6,1.4 \mathrm{~Hz}, 1 \mathrm{H})$, $7.59(\mathrm{~d}, J=3.8 \mathrm{~Hz}, 1 \mathrm{H}), 7.58(\mathrm{~s}, 1 \mathrm{H}), 7.34(\mathrm{dd}, J=6.3,2.6 \mathrm{~Hz}, 1 \mathrm{H}), 7.26(\mathrm{~d}, J=7.8 \mathrm{~Hz}, 1 \mathrm{H}), 5.03(\mathrm{~s}, 1 \mathrm{H})$, $3.83(\mathrm{~s}, 3 \mathrm{H}) ;{ }^{13} \mathrm{C} \mathrm{NMR}\left(100 \mathrm{MHz}, \mathrm{CDCl}_{3}\right) \delta 168.9,159.6,141.9,140.2,138.1,135.3,129.8,129.4,129.2$, 127.3, 126.5, 123.0, 54.1, 47.7; IR (film): 3059, 1645, 1326, 1235, 1044, $612 \mathrm{~cm}^{-1}$; HRMS (EI): $\mathrm{m} / z$ calcd for $\mathrm{C}_{14} \mathrm{H}_{11} \mathrm{BrN}_{2} \mathrm{O}_{2} \mathrm{~S}$ : 349.9725 ; found: 349.9726

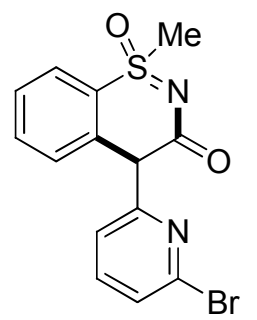

$4 a$

4-(6-Bromopyridin-2-yl)-1,8-dimethylbenzo[e][1,2]thiazin-3(4H)-one 1-oxide (4b): Yield : $61.9 \mathrm{mg}$ $(85 \%) R_{f}=0.3$ (EtOAc: $\left.\mathrm{CH}_{2} \mathrm{Cl}_{2}=1: 1\right)$; Pale yellow solid; Melting point: $182-184{ }^{\circ} \mathrm{C} ;{ }^{1} \mathrm{H} \mathrm{NMR}(400 \mathrm{MHz}$, $\left.\mathrm{CDCl}_{3}\right) \delta 7.59-7.52(\mathrm{~m}, 2 \mathrm{H}), 7.48(\mathrm{~d}, J=7.6 \mathrm{~Hz}, 1 \mathrm{H}), 7.36(\mathrm{~d}, J=7.6 \mathrm{~Hz}, 1 \mathrm{H}), 7.32(\mathrm{dd}, J=7.5,1.3 \mathrm{~Hz}$ $1 \mathrm{H}), 7.08(\mathrm{~d}, J=7.7 \mathrm{~Hz} 1 \mathrm{H}), 5.00(\mathrm{~s}, 1 \mathrm{H}), 3.86(\mathrm{~s}, 3 \mathrm{H}), 2.85(\mathrm{~s}, 3 \mathrm{H}) ;{ }^{13} \mathrm{C} \mathrm{NMR}\left(100 \mathrm{MHz}, \mathrm{CDCl}_{3}\right) \delta$ $168.3,159.9,141.8,140.2,138.9,138.6,134.5,132.6,128.0,127.7,127.2$, 122.9, 54.8, 46.6, 21.0; IR (film): 3059, 1648, 1334, 1227, 1046, $619 \mathrm{~cm}^{-1}$; HRMS (EI): $\mathrm{m} / z$ calcd for $\mathrm{C}_{15} \mathrm{H}_{13} \mathrm{BrN}_{2} \mathrm{O}_{2} \mathrm{~S}: 363.9881$; found: 363.9883

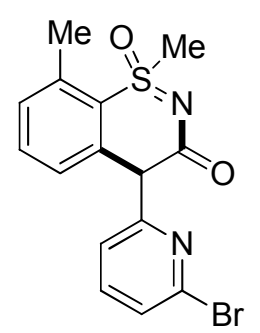


4-(6-Chloro-4-methylpyridin-2-yl)-1,8-dimethylbenzo[e][1,2] thiazin-3(4H)-one 1-oxide (4c): Yield : $46.8 \mathrm{mg}(70 \%) R_{f}=0.4$ (EtOAc: $\mathrm{CH}_{2} \mathrm{Cl}_{2}=1: 1$ ); Pale yellow solid; Melting point: $247-249{ }^{\circ} \mathrm{C}$; ${ }^{1} \mathrm{HNMR}$ $\left(400 \mathrm{MHz}, \mathrm{CDCl}_{3}\right) \delta 7.48(\mathrm{t}, J=7.7 \mathrm{~Hz}, 1 \mathrm{H}), 7.34(\mathrm{~d}, J=7.6 \mathrm{~Hz}, 1 \mathrm{H}), 7.31(\mathrm{~s}, 1 \mathrm{H}), 7.07$ (d, $J=7.6 \mathrm{~Hz}$, $1 \mathrm{H}), 6.98(\mathrm{~s}, 1 \mathrm{H}), 4.94(\mathrm{~s}, 1 \mathrm{H}), 3.87(\mathrm{~s}, 3 \mathrm{H}), 2.84(\mathrm{~s}, 3 \mathrm{H}), 2.36(\mathrm{~s}, 3 \mathrm{H}) ;{ }^{13} \mathrm{C} \mathrm{NMR}\left(100 \mathrm{MHz}, \mathrm{CDCl}_{3}\right) \delta$ $168.6,158.8,152.5,151.1,139.1,138.5,134.4,132.5,127.9,127.7,123.9,123.6,54.8,46.5,21.0(2 \mathrm{C})$; IR (film): 3064, 1648, 1332, 1227, 1049, $737 \mathrm{~cm}^{-1}$; HRMS (EI): $m / z$ calcd for $\mathrm{C}_{16} \mathrm{H}_{15} \mathrm{ClN}_{2} \mathrm{O}_{2} \mathrm{~S}: 334.0543$; found: 334.0540

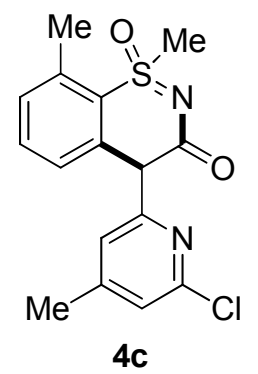




\section{Studies with isotopically labeled compounds}<smiles>[2H]c1ccccc1S(N)(=O)=O</smiles>

$1 \mathrm{a}-\left[D_{1}\right]$<smiles>CCOC(=O)c1nnn2c(Cl)cccc12</smiles>

2a

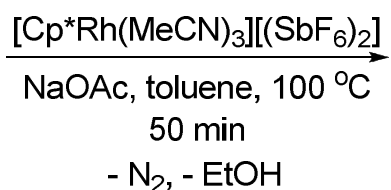

$k_{\mathrm{H}} / k_{\mathrm{D}}=3.0$<smiles>CS1(=O)=NC(=O)C(c2cccc(Cl)n2)c2cccc(O)c21</smiles>

$3 a+3 a-\left[D_{1}\right]$ $50 \%$

To a screw-top V-vial, sulfoximine- $D_{1} \quad\left(\mathbf{1 a}_{\mathbf{a}}\left[\mathbf{D}_{\mathbf{1}}\right]\right)$ (1.0 equiv, $\left.0.2 \mathrm{mmol}\right)$, ethyl 7-chloro$[1,2,3]$ triazolo[1,5-a]pyridine-3-carboxylate (2a) (1.0 equiv, $0.2 \mathrm{mmol}),\left[\mathrm{Cp} * \mathrm{Rh}(\mathrm{MeCN})_{3}\right]\left[\mathrm{SbF}_{6}\right]_{2}(3$ mol \%), and $\mathrm{NaOAc}(1.0$ equiv, $0.2 \mathrm{mmol})$ in toluene $(2.0 \mathrm{~mL})$ were added. The resulting mixture was stirred under air at $100{ }^{\circ} \mathrm{C}$ for $50 \mathrm{~min}$. After celite filtration and evaporation of the solvents in vacuo, the crude product was purified by column chromatography on silica gel using EtOAc: $\mathrm{CH}_{2} \mathrm{Cl}_{2}=1: 1$ to yield $3 \mathbf{a}+\mathbf{3 a}-\left[\mathbf{D}_{1}\right](50 \%)$ as a ivory solid. The ratio of two products was determined by ${ }^{1} \mathrm{H}$ NMR integration method to give intramolecular kinetic isotopic effect (KIE) $k_{\mathrm{H}} / k_{\mathrm{D}}=3.0$.

\section{Competition experiments}

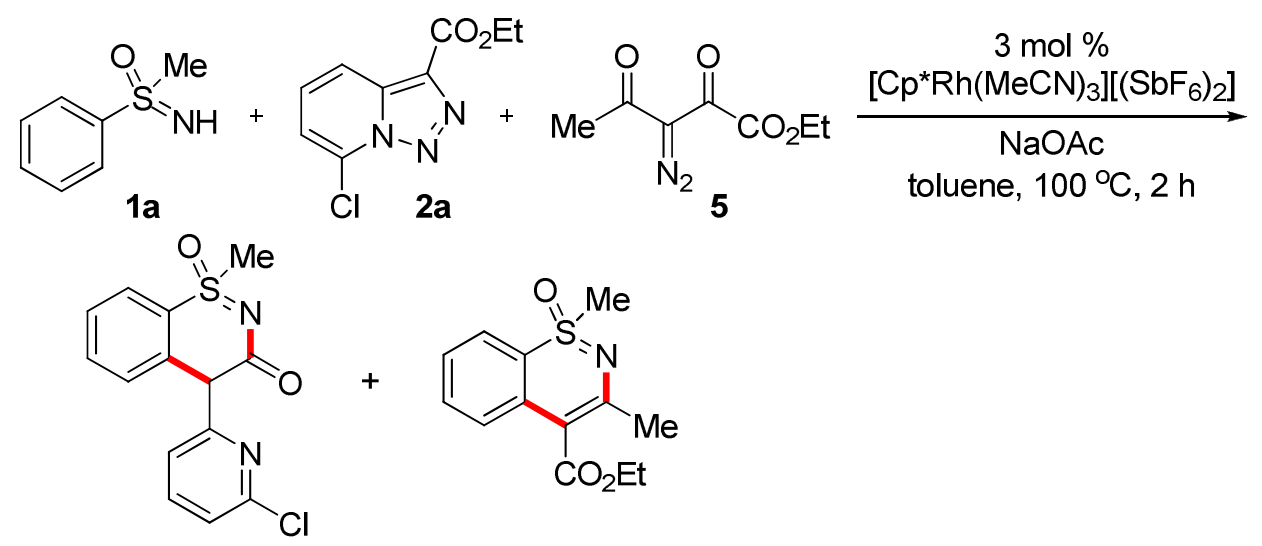

$3 a, 62 \%$

$6,26 \%$

Following the general procedure, the $\mathrm{C}-\mathrm{H}$ activation/cyclization/elimination reaction was carried out with $S$-phenylsulfoximine (1a) (1.0 equiv, $0.2 \mathrm{mmol})$, ethyl 7-chloro-[1,2,3]triazolo[1,5-a]pyridine-3carboxylate (2a) (1.0 equiv, $0.2 \mathrm{mmol}$ ), ethyl 3-diazo-2,4-dioxopentanoate (5) (1.0 equiv, $0.2 \mathrm{mmol}$ ), $\left[\mathrm{Cp} * \mathrm{Rh}(\mathrm{MeCN})_{3}\right]\left[\mathrm{SbF}_{6}\right]_{2}(3 \mathrm{~mol} \%$ ), and $\mathrm{NaOAc}(1.0$ equiv, $0.2 \mathrm{mmol})$ in toluene $(2.0 \mathrm{~mL})$. The resulting mixture was stirred at $100{ }^{\circ} \mathrm{C}$ for $2 \mathrm{~h}$ under argon. The reaction mixture was analyzed using NMR analysis. 
<smiles></smiles>

$1 \mathrm{~g}$

11

$\mathrm{Me}$<smiles>COc1ccc2c(c1)C(c1cccc(Cl)n1)C(=O)N=S2(C)=O</smiles>

$3 \mathrm{~g}, 51 \%$<smiles>CS1(=O)=NC(=O)C(c2cccc(Cl)n2)c2cc(Br)ccc21</smiles>

3I, $35 \%$

Following the general procedure, the $\mathrm{C}-\mathrm{H}$ activation/cyclization/elimination reaction was carried out with $S$-(4-methoxy)phenyl-S-methylsulfoximine (1g) (1.0 equiv, $0.2 \mathrm{mmol}), S$-(4-bromo)phenyl-Smethylsulfoximine (11) (1.0 equiv, $0.2 \mathrm{mmol}$ ), ethyl 7-chloro-[1,2,3]triazolo[1,5-a]pyridine-3-carboxylate (2a) (1.0 equiv, $0.2 \mathrm{mmol})$, $\left[\mathrm{Cp} * \mathrm{Rh}(\mathrm{MeCN})_{3}\right]\left[\mathrm{SbF}_{6}\right]_{2}(3 \mathrm{~mol} \%)$, and $\mathrm{NaOAc}(1.0$ equiv, $0.2 \mathrm{mmol})$ in toluene $(2.0 \mathrm{~mL})$. The resulting mixture was stirred at $100{ }^{\circ} \mathrm{C}$ for $2 \mathrm{~h}$ under argon. The reaction mixture was analyzed using NMR analysis. 


\section{X-ray crystallography of 3a}

A colorless block-like specimen of $\mathrm{C}_{14} \mathrm{H}_{11} \mathrm{ClN}_{2} \mathrm{O}_{2} \mathrm{~S}$, approximate dimensions $0.100 \mathrm{~mm} \times 0.240 \mathrm{~mm} \times$ $0.360 \mathrm{~mm}$, was used for the X-ray crystallographic analysis. The X-ray intensity data were measured. The X-ray intensity data were measured. Structural information was deposited at the Cambridge Crystallographic Data Center (CCDC 1478952).

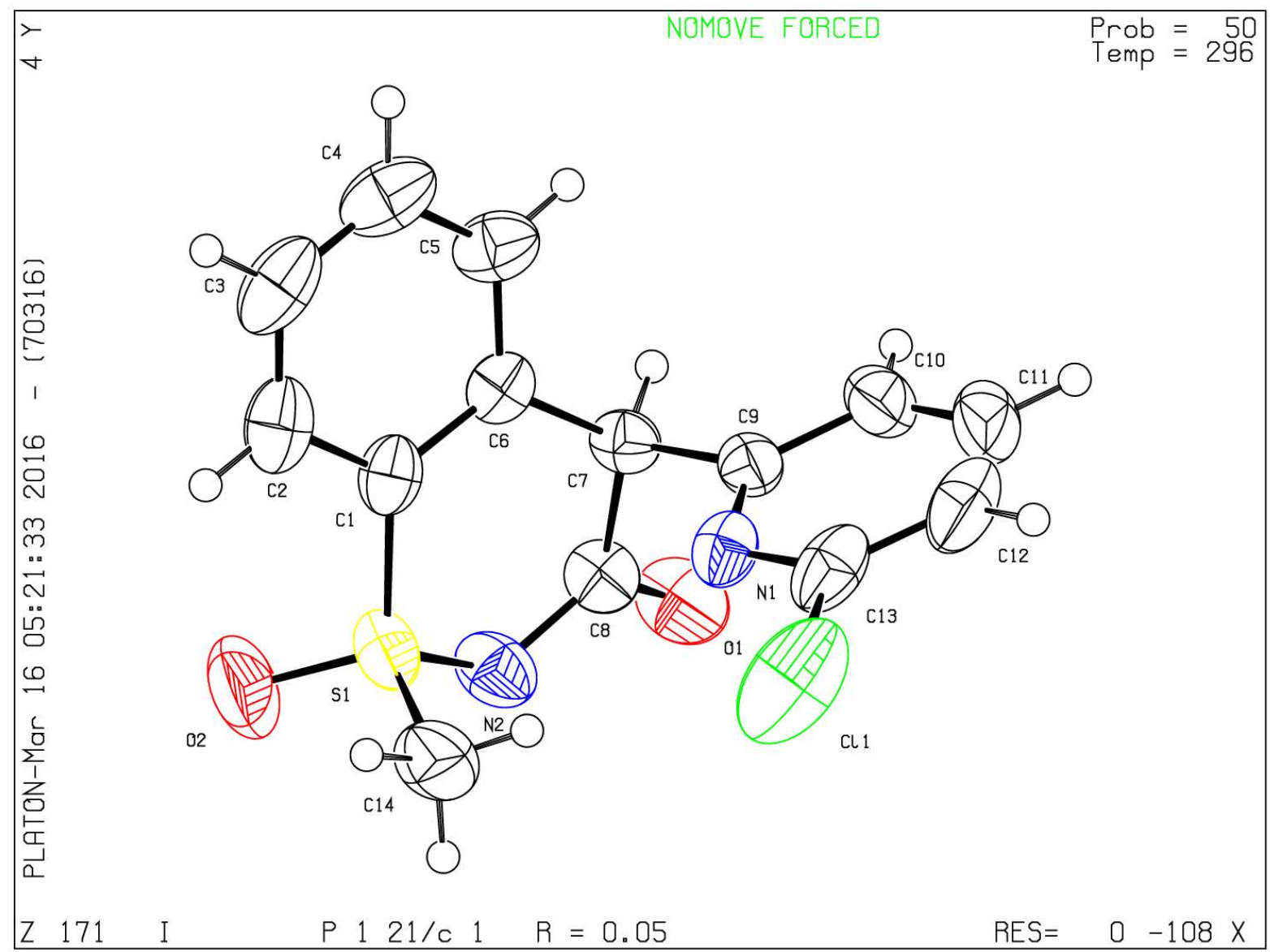

Table 2. Sample and crystal data for 3a.

\begin{tabular}{|l|l||}
\hline Identification code & $\mathbf{3 a}$ \\
\hline \hline Chemical formula & $\mathrm{C}_{14} \mathrm{H}_{11} \mathrm{ClN}_{2} \mathrm{O}_{2} \mathrm{~S}$ \\
\hline Formula weight & 306.76 \\
\hline \hline Temperature & $296(2) \mathrm{K}$ \\
\hline Wavelength & $0.71073 \AA$ \\
\hline \hline Crystal size & $0.100 \times 0.240 \times 0.360 \mathrm{~mm}$ \\
\hline Crystal habit & colorless block \\
\hline Crystal system & monoclinic \\
\hline \hline Space group & $\mathrm{P} 122_{1} / \mathrm{c} 1$ \\
\hline \hline Unit cell dimensions & $\mathrm{a}=15.5476(9) \AA$ \\
\hline \hline & $\mathrm{b}=10.8589(9) \AA$ \\
\hline
\end{tabular}




\begin{tabular}{||l|l|l|}
\hline & $\mathrm{c}=8.1418(5) \AA$ & $\gamma=90^{\circ}$ \\
\hline Volume & $1373.48(16) \AA^{3}$ & \\
\hline $\mathbf{Z}$ & 4 \\
\hline Density (calculated) & $1.483 \mathrm{~g} / \mathrm{cm}^{3}$ \\
\hline \hline Absorption coefficient & $0.432 \mathrm{~mm}^{-1}$ \\
\hline F(000) & 632 \\
\hline
\end{tabular}

Table 2. Data collection and structure refinement for $\mathbf{3 a}$.

\begin{tabular}{|c|c|c|}
\hline Theta range for data collection & \multicolumn{2}{|l|}{1.31 to $28.42^{\circ}$} \\
\hline Index ranges & \multicolumn{2}{|c|}{$-20<=\mathrm{h}<=20,-14<=\mathrm{k}<=14,-10<=\mathrm{l}<=10$} \\
\hline Reflections collected & \multicolumn{2}{|l|}{43701} \\
\hline Independent reflections & \multicolumn{2}{|c|}{$3446[\mathrm{R}(\mathrm{int})=0.0501]$} \\
\hline Coverage of independent reflections & \multicolumn{2}{|l|}{$99.9 \%$} \\
\hline Absorption correction & \multicolumn{2}{|l|}{ multi-scan } \\
\hline Max. and min. transmission & \multicolumn{2}{|l|}{0.9580 and 0.8600} \\
\hline Refinement method & \multicolumn{2}{|c|}{ Full-matrix least-squares on $\mathrm{F}^{2}$} \\
\hline Refinement program & \multicolumn{2}{|c|}{ SHELXL-2013 (Sheldrick, 2013) } \\
\hline Function minimized & \multicolumn{2}{|l|}{$\sum \mathrm{w}\left(\mathrm{F}_{\mathrm{o}}^{2}-\mathrm{F}_{\mathrm{c}}^{2}\right)^{2}$} \\
\hline Data / restraints / parameters & \multicolumn{2}{|l|}{$3446 / 0 / 182$} \\
\hline Goodness-of-fit on $\mathrm{F}^{2}$ & \multicolumn{2}{|l|}{1.055} \\
\hline \multirow[t]{2}{*}{ Final $\mathbf{R}$ indices } & 2439 data; $\mathrm{I}>2 \sigma(\mathrm{I})$ & $\mathrm{R} 1=0.0502, \mathrm{wR} 2=0.1191$ \\
\hline & all data & $\mathrm{R} 1=0.0763, \mathrm{wR} 2=0.1329$ \\
\hline Weighting scheme & \multicolumn{2}{|c|}{$\begin{array}{l}\mathrm{w}=1 /\left[\sigma^{2}\left(\mathrm{~F}_{\mathrm{o}}^{2}\right)+(0.0509 \mathrm{P})^{2}+0.7599 \mathrm{P}\right] \\
\text { where } \mathrm{P}=\left(\mathrm{F}_{\mathrm{o}}{ }^{2}+2 \mathrm{~F}_{\mathrm{c}}^{2}\right) / 3\end{array}$} \\
\hline Largest diff. peak and hole & \multicolumn{2}{|c|}{0.313 and $-0.522 \mathrm{e}^{-3}$} \\
\hline R.M.S. deviation from mean & \multicolumn{2}{|l|}{$0.047 \mathrm{e}^{-3}$} \\
\hline
\end{tabular}

Table 3. Bond lengths (Å) for 3a.

\begin{tabular}{|l|l|l|l||}
\hline C1-C6 & $1.386(3)$ & C1-C2 & $1.396(3)$ \\
\hline C1-S1 & $1.752(2)$ & C2-C3 & $1.371(4)$ \\
\hline C2-H2 & 0.93 & C3-C4 & $1.379(4)$ \\
\hline C3-H3 & 0.93 & C4-C5 & $1.373(4)$ \\
\hline C4-H4 & 0.93 & C5-C6 & $1.389(3)$ \\
\hline C5-H5 & 0.93 & C6-C7 & $1.506(3)$ \\
\hline C7-C9 & $1.512(3)$ & C7-C8 & $1.525(3)$ \\
\hline \hline C7-H7 & 0.98 & C8-O1 & $1.212(3)$ \\
\hline C8-N2 & $1.362(3)$ & C9-N1 & $1.345(3)$ \\
\hline C9-C10 & $1.378(3)$ & C10-C11 & $1.371(4)$ \\
\hline C10-H10 & 0.93 & C11-C12 & $1.361(5)$ \\
\hline \hline C11-H11 & 0.93 & C12-C13 & $1.392(4)$ \\
\hline C12-H12 & 0.93 & C13-N1 & $1.317(3)$ \\
\hline C13-C11 & $1.736(3)$ & C14-S1 & $1.750(3)$ \\
\hline \hline C14-H14A & 0.96 & C14-H14B & 0.96 \\
\hline C14-H14C & 0.96 & N2-S1 & $1.543(2)$ \\
\hline \hline O2-S1 & $1.4386(19)$ & \\
\hline \hline
\end{tabular}


Table 4. Bond angles $\left({ }^{\circ}\right)$ for $3 a$.

\begin{tabular}{|l||l|l|l||}
\hline C6-C1-C2 & $122.0(2)$ & C6-C1-S1 & $118.30(16)$ \\
\hline C2-C1-S1 & $119.60(19)$ & C3-C2-C1 & $118.8(3)$ \\
\hline C3-C2-H2 & 120.6 & C1-C2-H2 & 120.6 \\
\hline C2-C3-C4 & $120.2(2)$ & C2-C3-H3 & 119.9 \\
\hline C4-C3-H3 & 119.9 & C5-C4-C3 & $120.5(3)$ \\
\hline \hline C5-C4-H4 & 119.8 & C3-C4-H4 & 119.8 \\
\hline C4-C5-C6 & $121.2(3)$ & C4-C5-H5 & 119.4 \\
\hline C6-C5-H5 & 119.4 & C1-C6-C5 & $117.3(2)$ \\
\hline C1-C6-C7 & $122.59(19)$ & C5-C6-C7 & $120.0(2)$ \\
\hline C6-C7-C9 & $109.30(16)$ & C6-C7-C8 & $116.96(18)$ \\
\hline C9-C7-C8 & $108.67(17)$ & C6-C7-H7 & 107.2 \\
\hline C9-C7-H7 & 107.2 & C8-C7-H7 & 107.2 \\
\hline O1-C8-N2 & $119.9(2)$ & O1-C8-C7 & $118.4(2)$ \\
\hline N2-C8-C7 & $121.65(19)$ & N1-C9-C10 & $122.2(2)$ \\
\hline N1-C9-C7 & $115.28(17)$ & C10-C9-C7 & $122.6(2)$ \\
\hline \hline C11-C10-C9 & $119.0(3)$ & C11-C10-H10 & 120.5 \\
\hline \hline C9-C10-H10 & 120.5 & C12-C11-C10 & $120.2(3)$ \\
\hline C12-C11-H11 & 119.9 & C10-C11-H11 & 119.9 \\
\hline C11-C12-C13 & $116.9(3)$ & C11-C12-H12 & 121.6 \\
\hline \hline C13-C12-H12 & 121.6 & N1-C13-C12 & $124.6(3)$ \\
\hline N1-C13-C11 & $115.3(2)$ & C12-C13-C11 & $120.2(2)$ \\
\hline S1-C14-H14A & 109.5 & S1-C14-H14B & 109.5 \\
\hline H14A-C14-H14B & 109.5 & S1-C14-H14C & 109.5 \\
\hline \hline H14A-C14-H14C & 109.5 & H14B-C14-H14C & 109.5 \\
\hline C13-N1-C9 & $117.2(2)$ & C8-N2-S1 \\
\hline O2-S1-N2 & $113.97(13)$ & O2-S1-C14 & $124.88(16)$ \\
\hline N2-S1-C14 & O2-S1-C1 \\
\hline N2-S1-C1 & C14-S1-C1 \\
\hline
\end{tabular}




\section{References}

(1) (a) Li, Y.; Li, B.-J.; Wang, W.-H.; Huang, W.-P.; Zhang, X.-S.; Chen, K.; Shi, Z.-J. Angew. Chem., Int. Ed. 2011, 50, 2115. (b) White, C.; Thompson, S. J.; Maitlis, P. M. J. C. S. Dalton, 1977, 1654.

(2) (a) Mancheno, O. G.; Bistri, O.; Bolm, C. Org. Lett. 2007, 9, 3809. (b) Okamura, H.; Bolm, C. Org. Lett. 2004, 6, 1305. (c) Joseph, P. J. A.; Priyadarshini, S.; Kantam, M. L.; Sreedhar, B. Tetrahedron, 2013, 69, 8276. (d) Yadav, M. R.; Rit, R. K.; Sashoo, A. K. Chem. Eur. J. 2012, 18, 5541.

(3) (a) Chuprakov, S.; Hwang, F. W.; Gevorgyan,V. Angew. Chem., Int. Ed. 2007, 46, 4757. (b) Shi, Y.; Gulevich, A. V.; Gevorgyan, V. Angew. Chem., Int. Ed. 2014, 53, 14191. (c) Kim, J. H.; Gensch, T.; Zhao, D.; Stegemann, L.; Strassert, C. A.; Glorius, F. Angew. Chem., Int. Ed. 2015, 54, 10975.

(4) (a) Chen, X.; Xie, Y.; Xiao, X.; Li, G.; Deng, Y.; Jiang, H.; Zheng, W. Chem. Commun. 2015, 51, 15328. (b) Jinag, Y.; Khong, V. Z. Y.; Lourdusamy, E.; Park, C.-M. Chem. Commun. 2012, 48, 3133. 


\section{8. ${ }^{1} \mathrm{H}$ and ${ }^{13} \mathrm{C}$ NMR spectra}
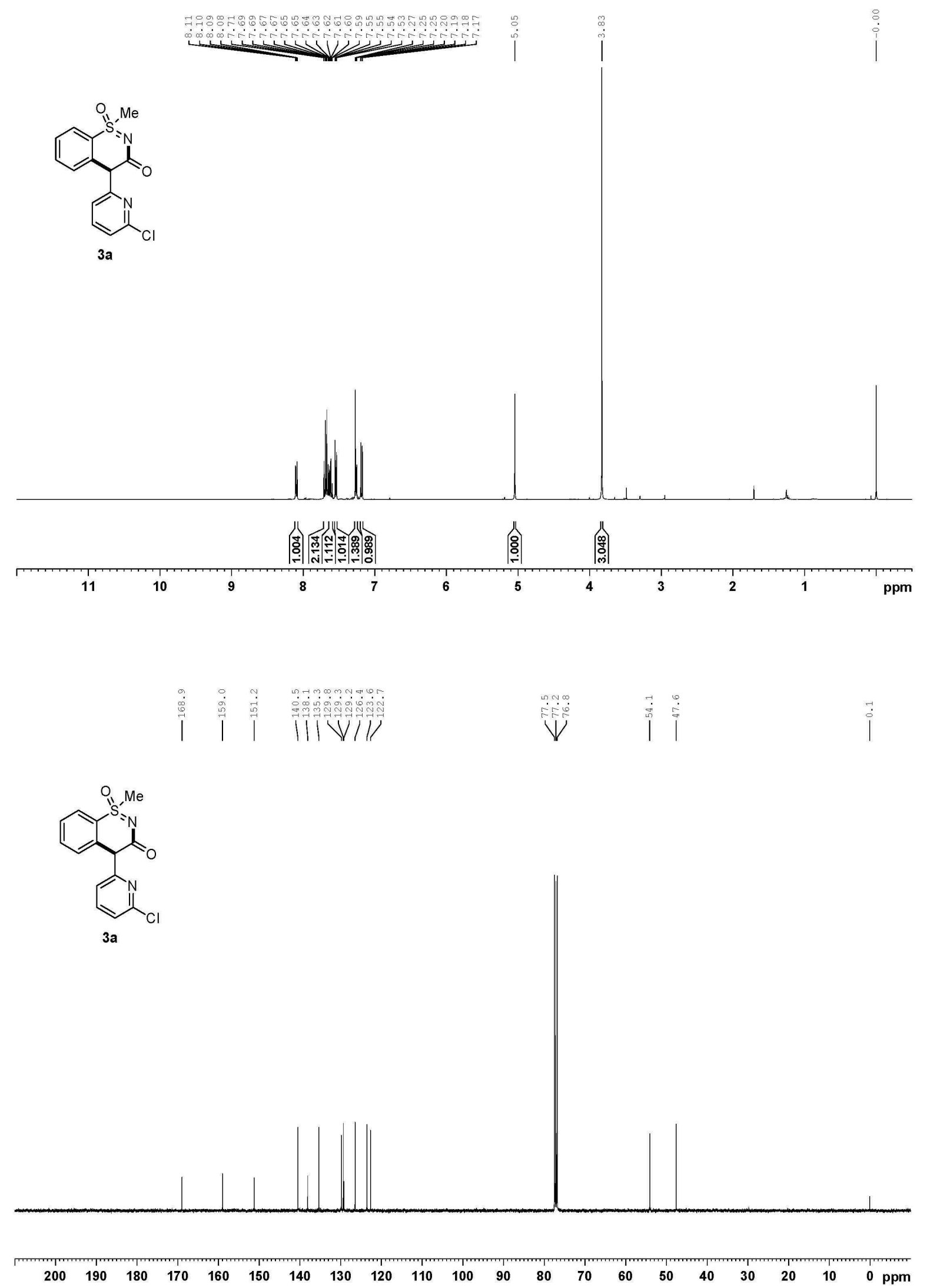

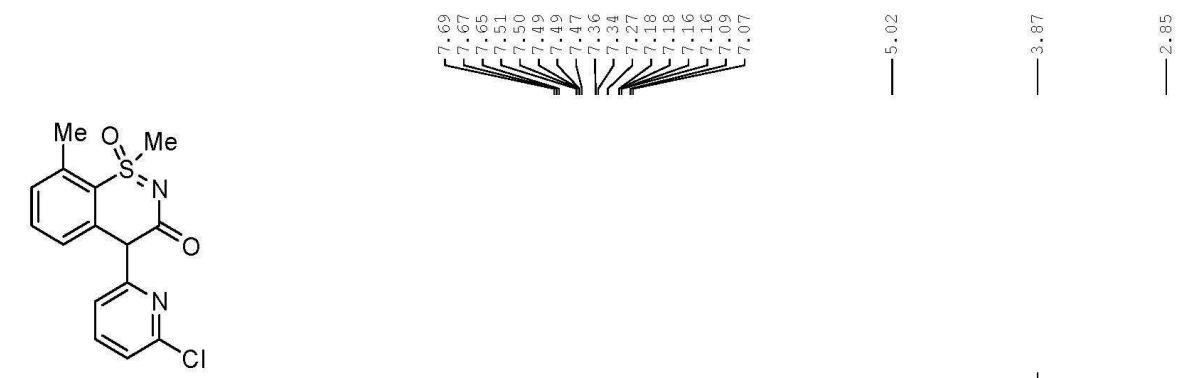

3b
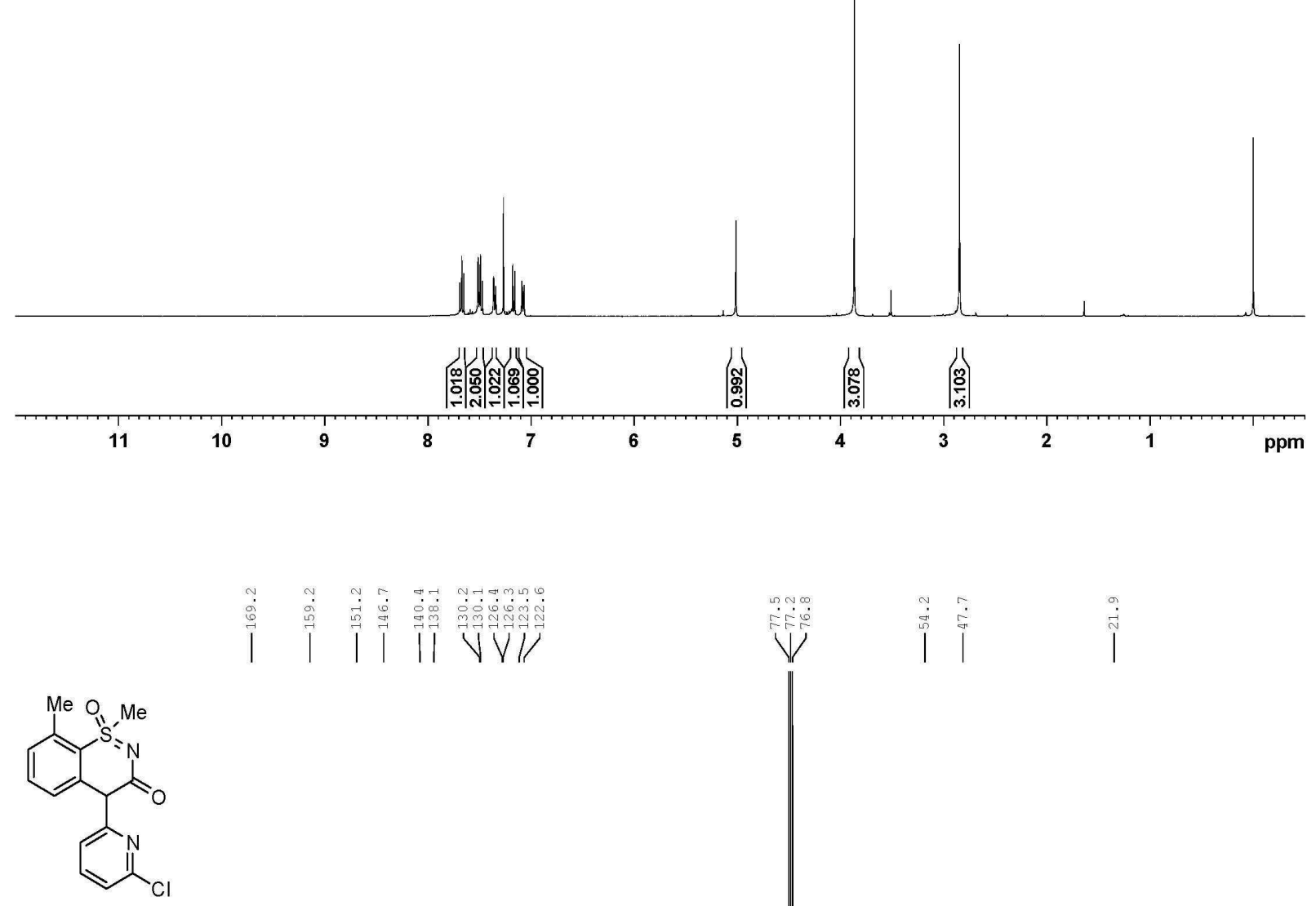

3b

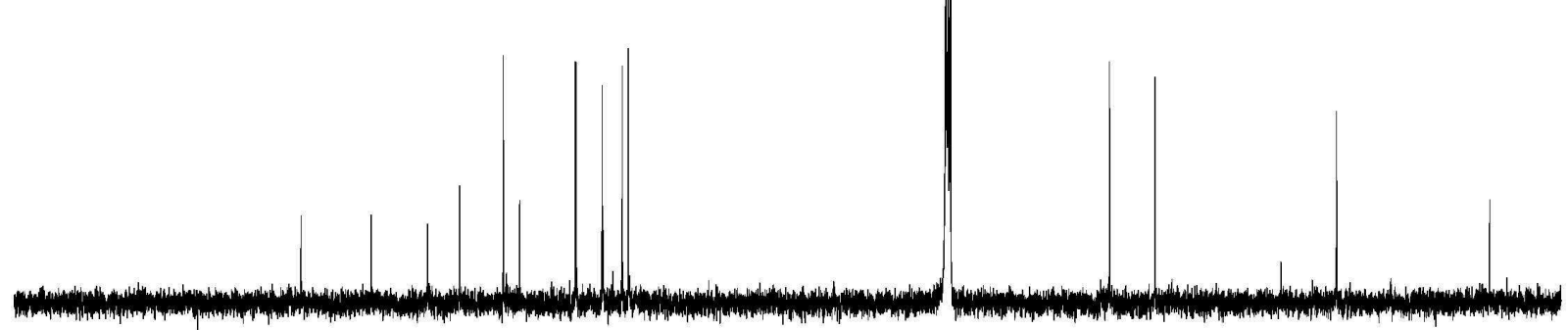

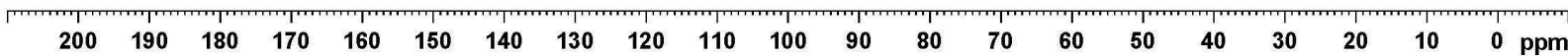



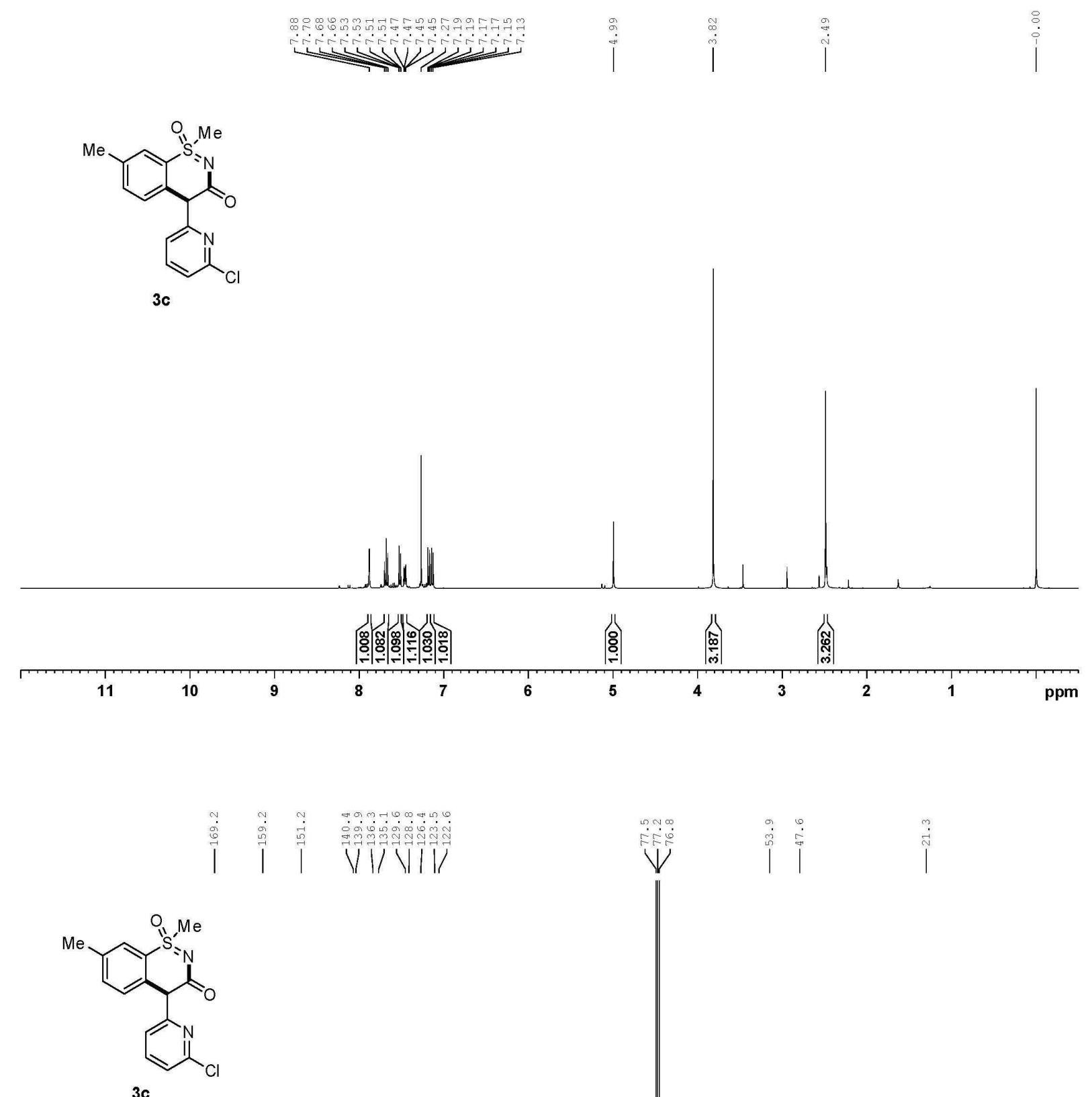

|
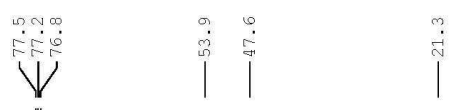

3c
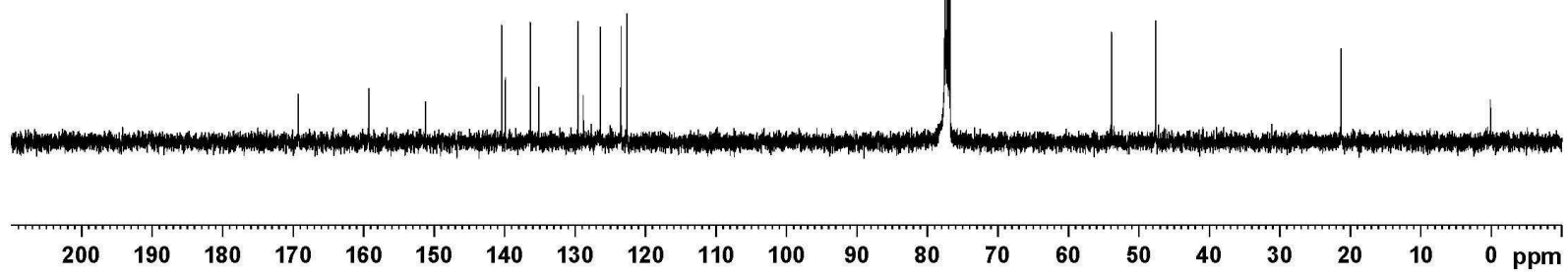


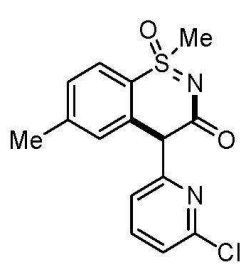

$3 d$
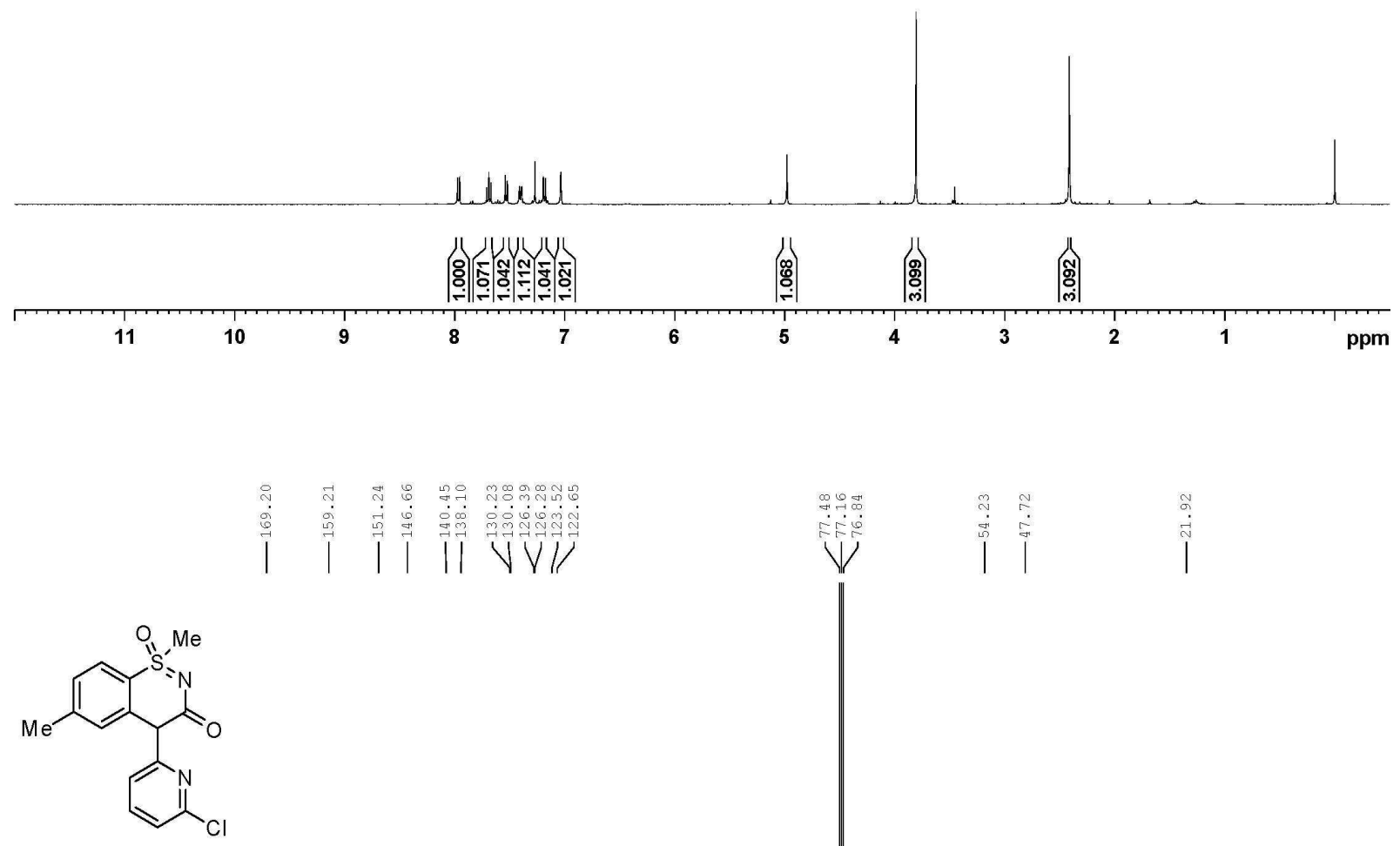

3d

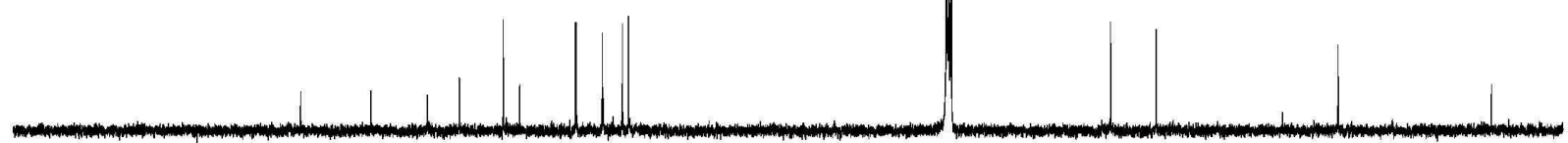

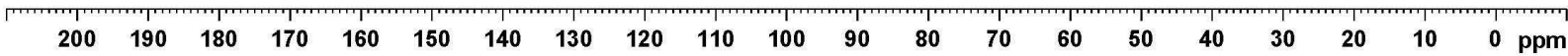



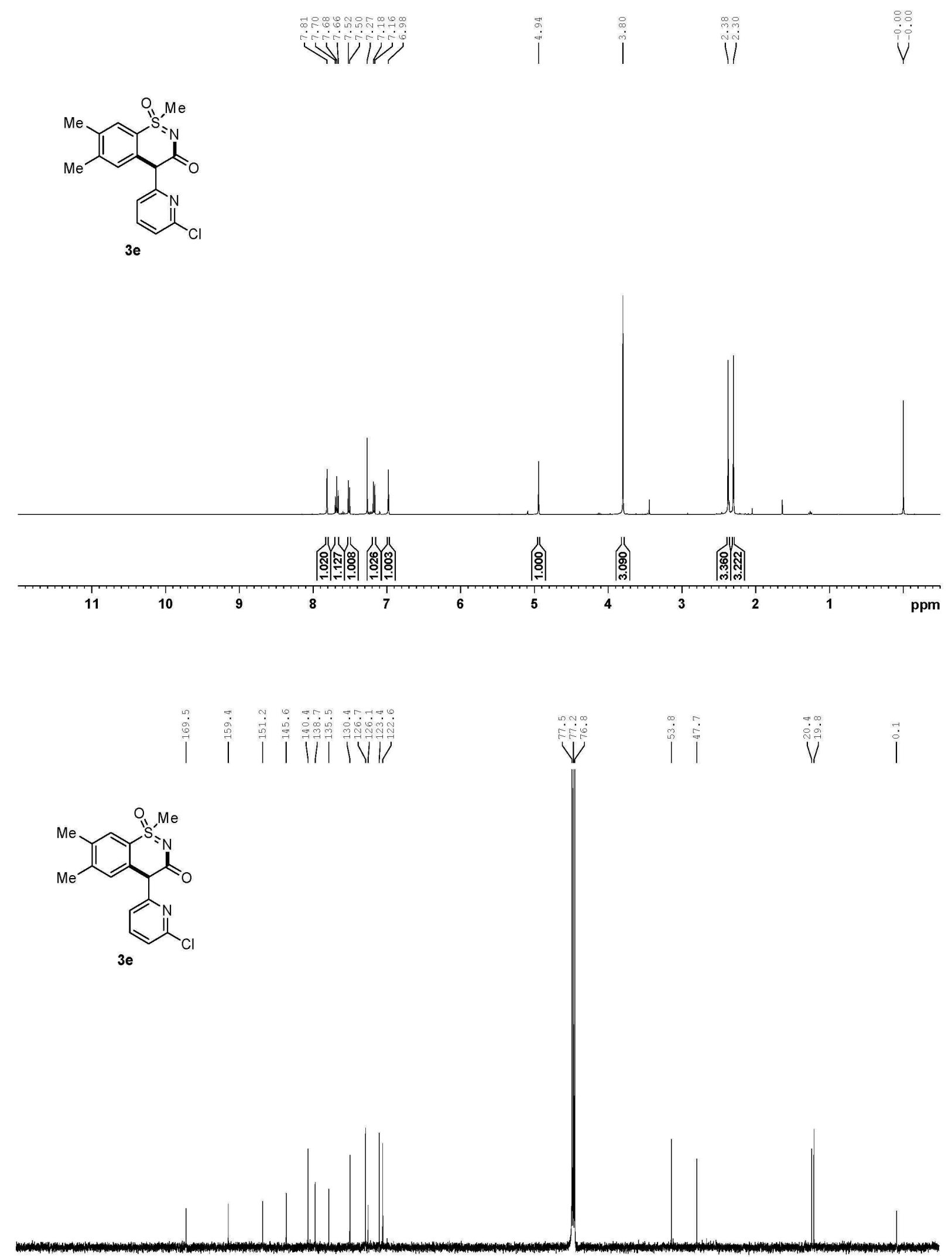

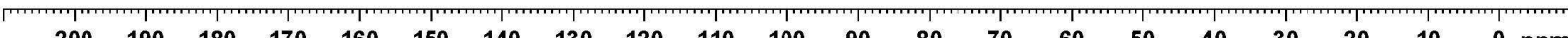



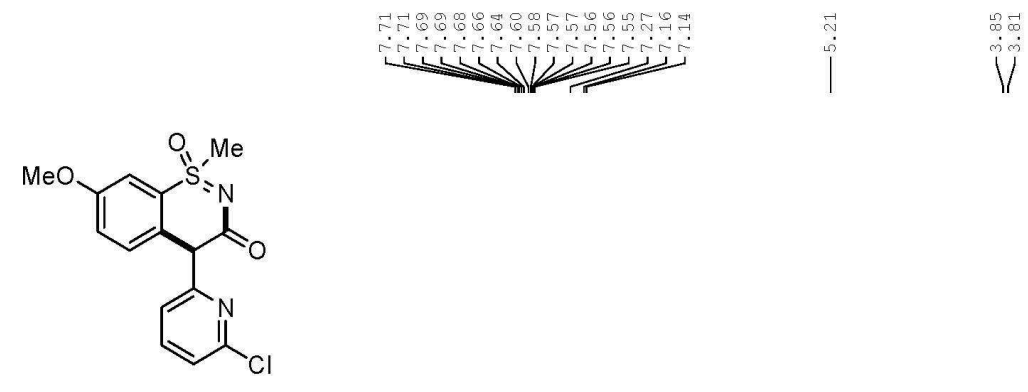

$3 \mathbf{f}$
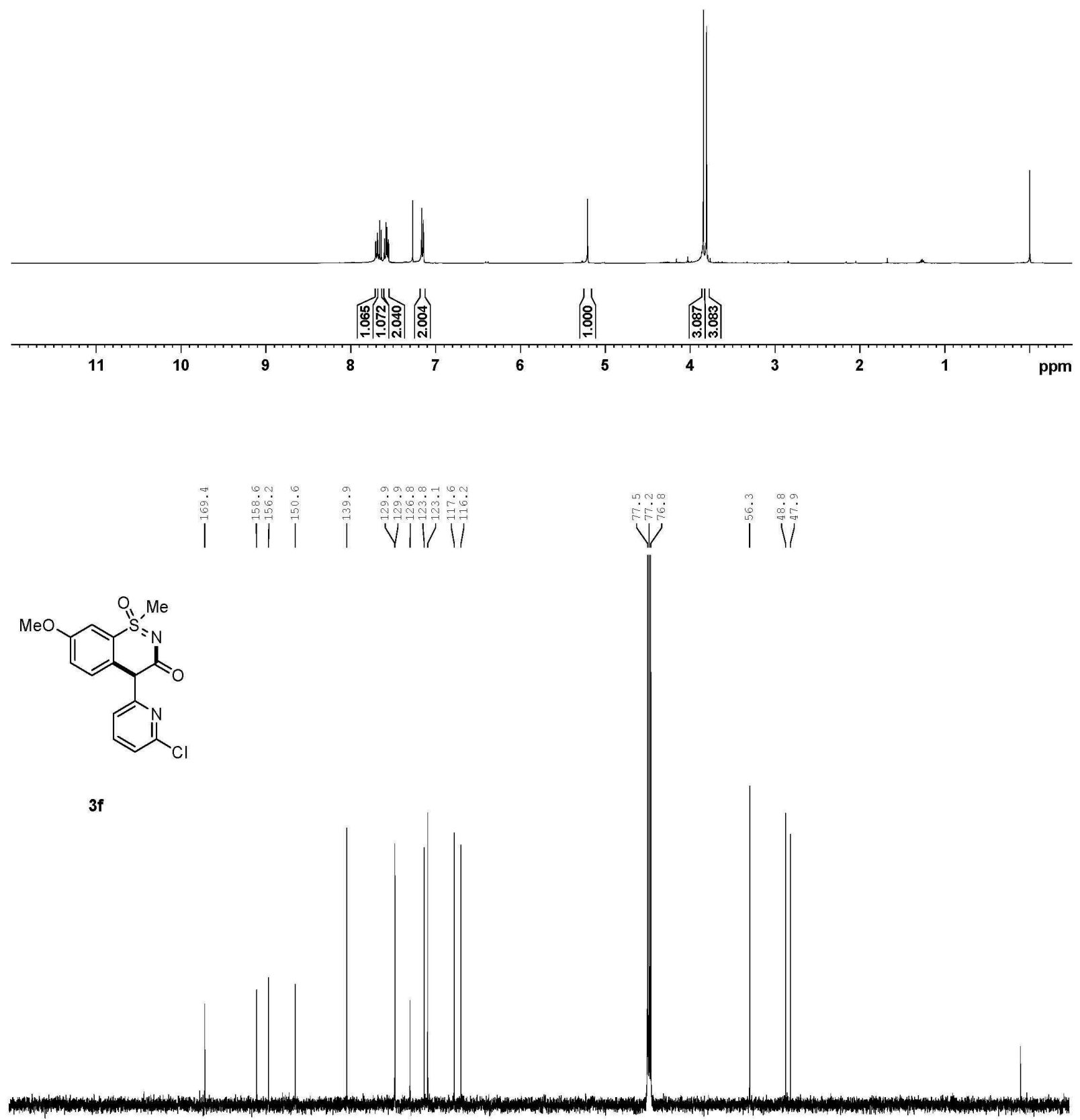

$\begin{array}{llllllllllllllllllllll}200 & 190 & 180 & 170 & 160 & 150 & 140 & 130 & 120 & 110 & 100 & 90 & 80 & 70 & 60 & 50 & 40 & 30 & 20 & 10 & 0 & \mathrm{ppm}\end{array}$ 


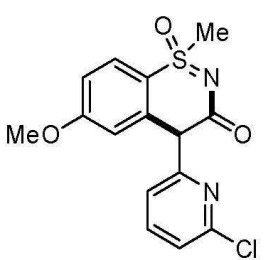

$3 \mathbf{g}$

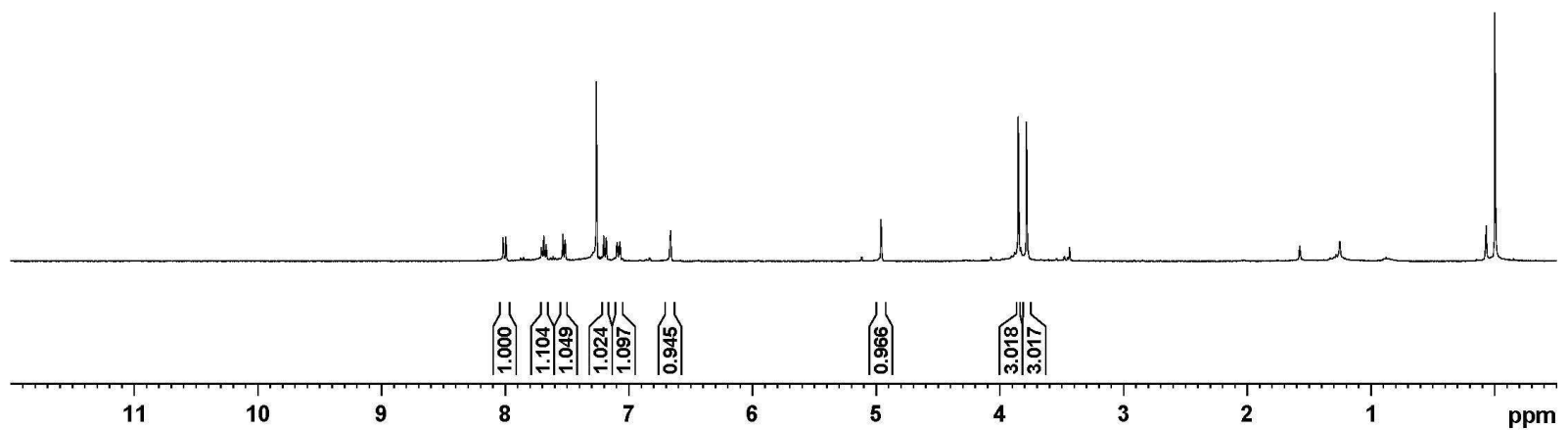

$$
\text { | | | | }
$$<smiles>COc1ccc2c(c1)C(c1cccc(Cl)n1)C(=O)N=S2(C)=O</smiles>

$3 \mathrm{~g}$

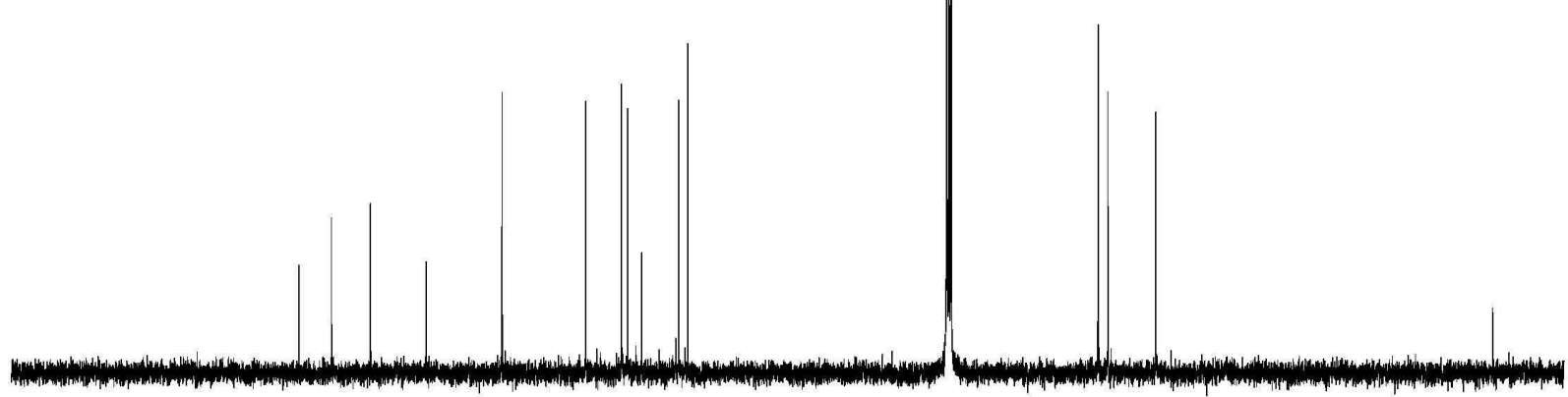

$\begin{array}{llllllllllllllllllllll}200 & 190 & 180 & 170 & 160 & 150 & 140 & 130 & 120 & 110 & 100 & 90 & 80 & 70 & 60 & 50 & 40 & 30 & 20 & 10 & 0 & \mathrm{ppm}\end{array}$ 

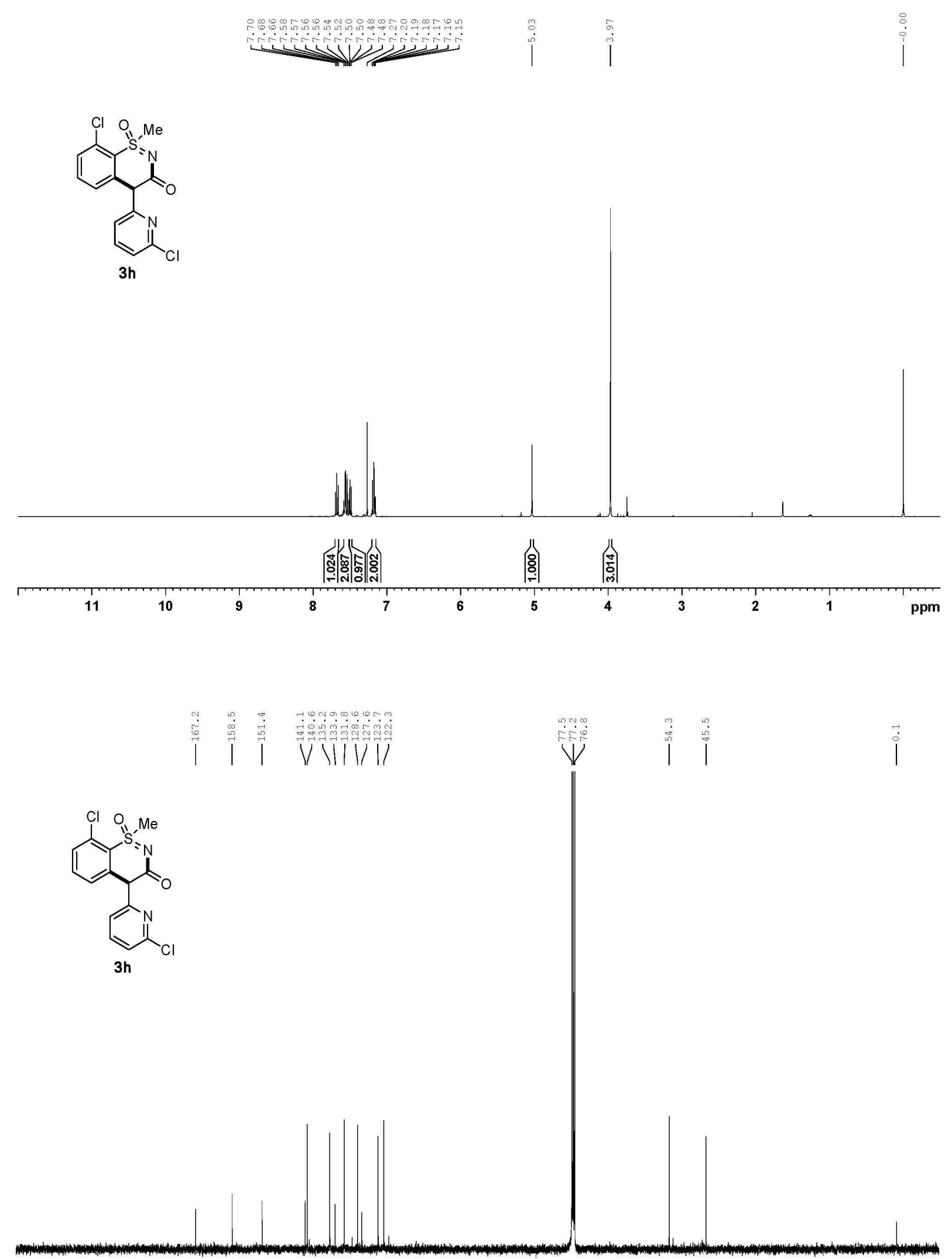

$\begin{array}{llllllllllllllllllllllllll}200 & 190 & 180 & 170 & 160 & 150 & 140 & 130 & 120 & 110 & 100 & 90 & 80 & 70 & 60 & 50 & 40 & 30 & 20 & 10 & 0 & \text { ppm }\end{array}$ 


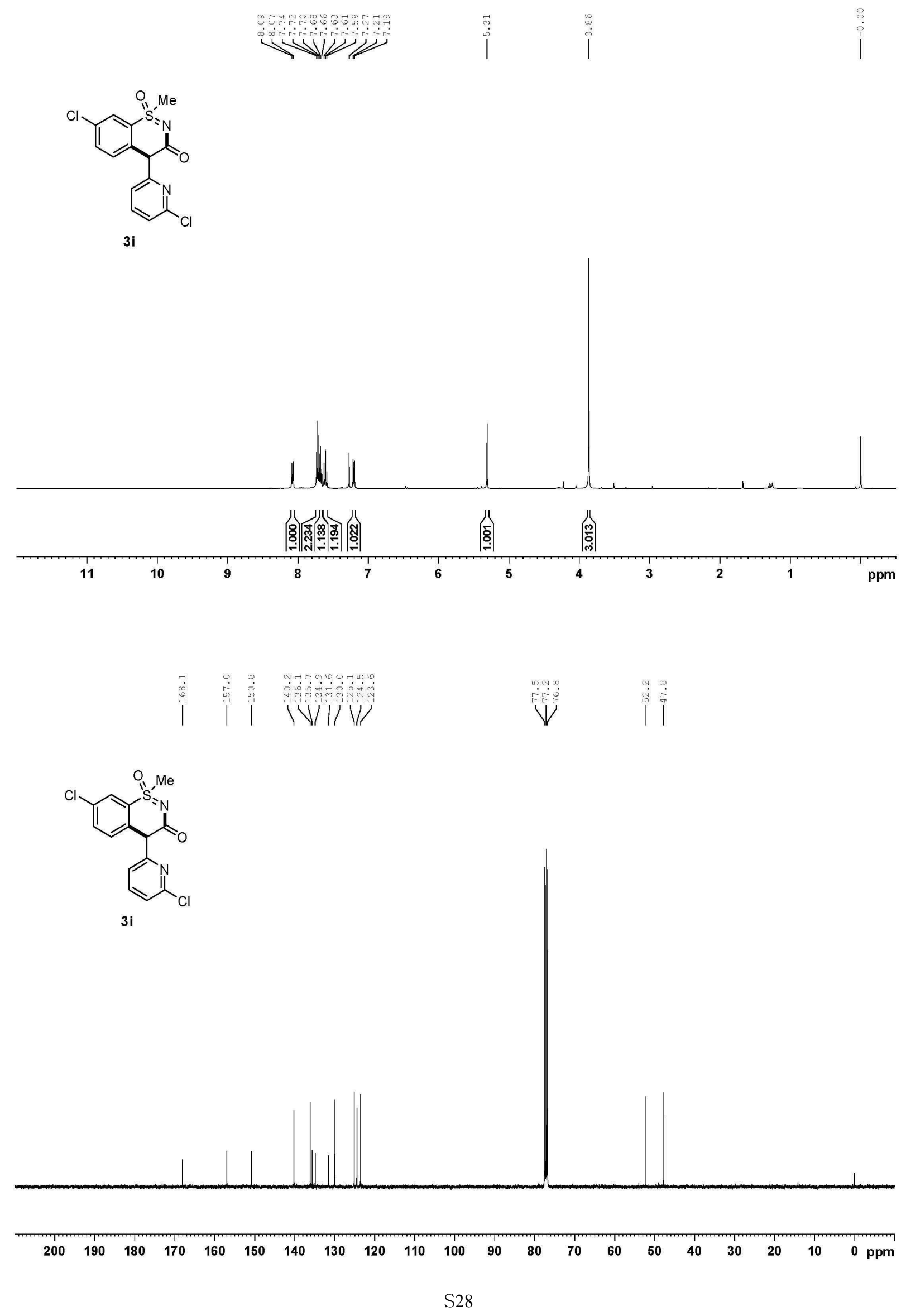



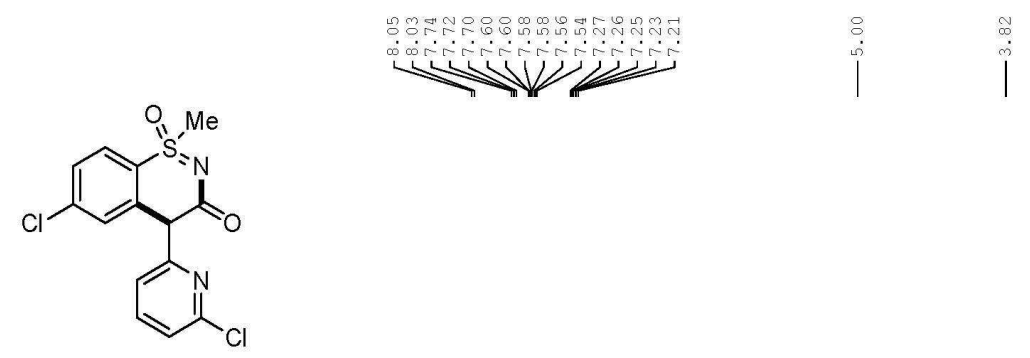

3j
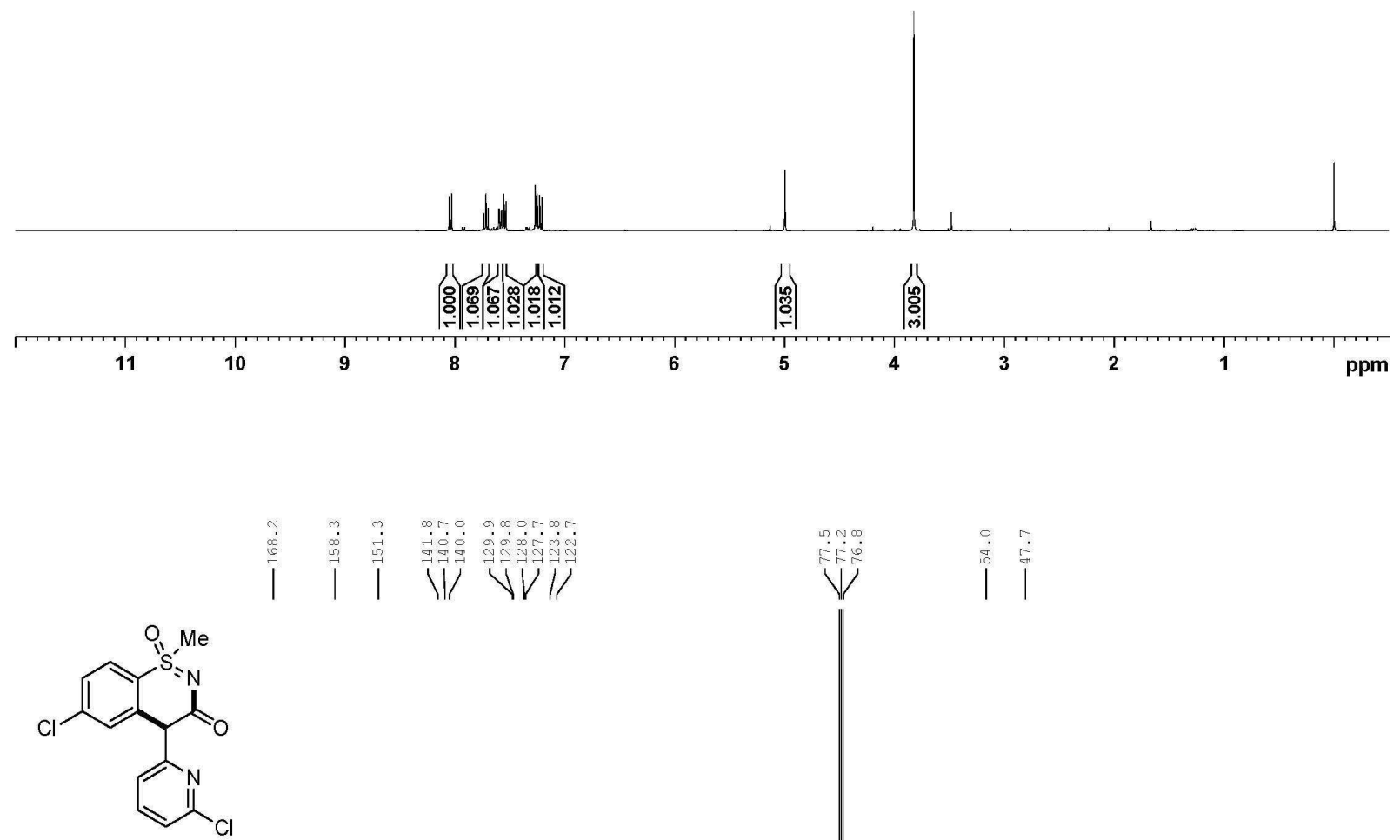

3j

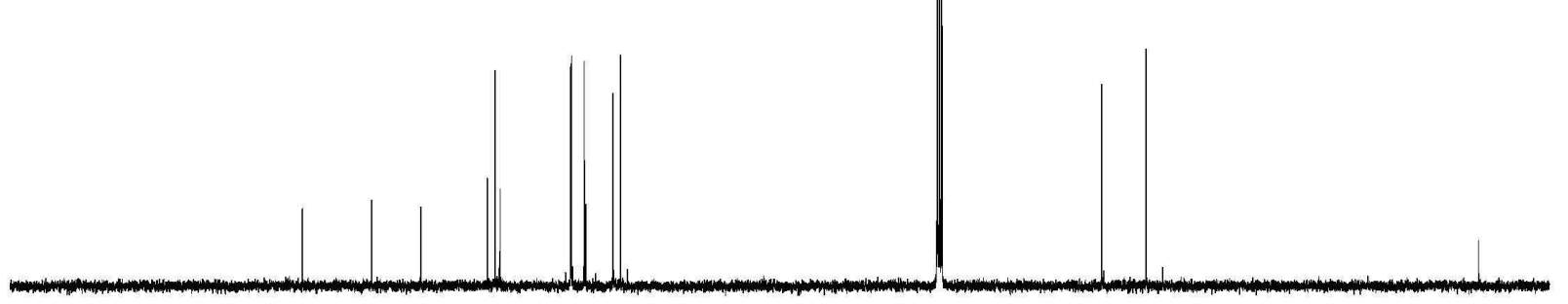

$\begin{array}{lllllllllllllllllllll}200 & 190 & 180 & 170 & 160 & 150 & 140 & 130 & 120 & 110 & 100 & 90 & 80 & 70 & 60 & 50 & 40 & 30 & 20 & 10 & 0\end{array}$ 

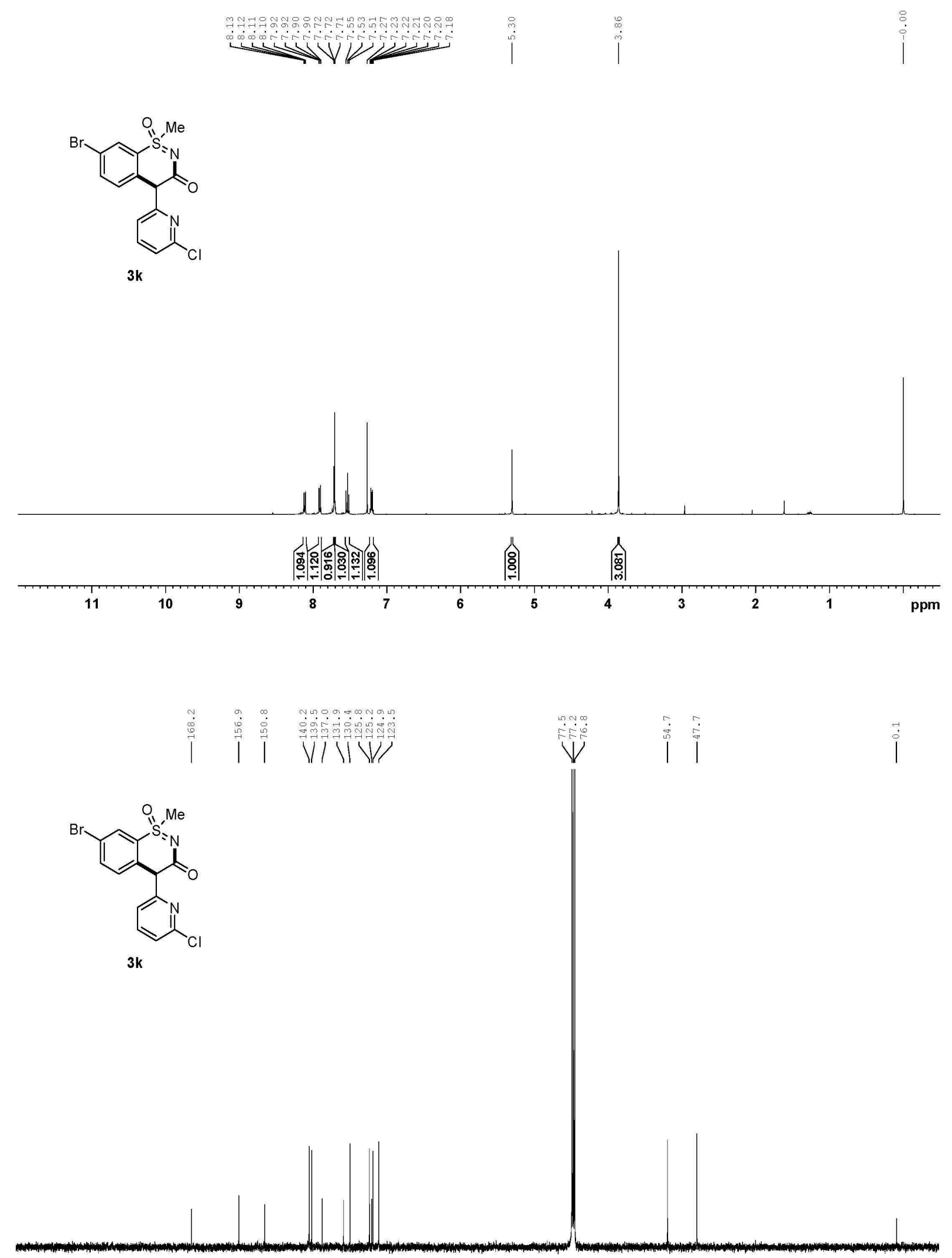

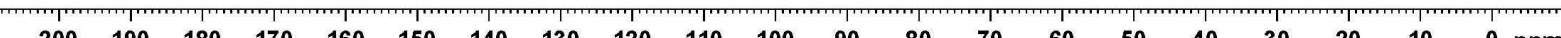



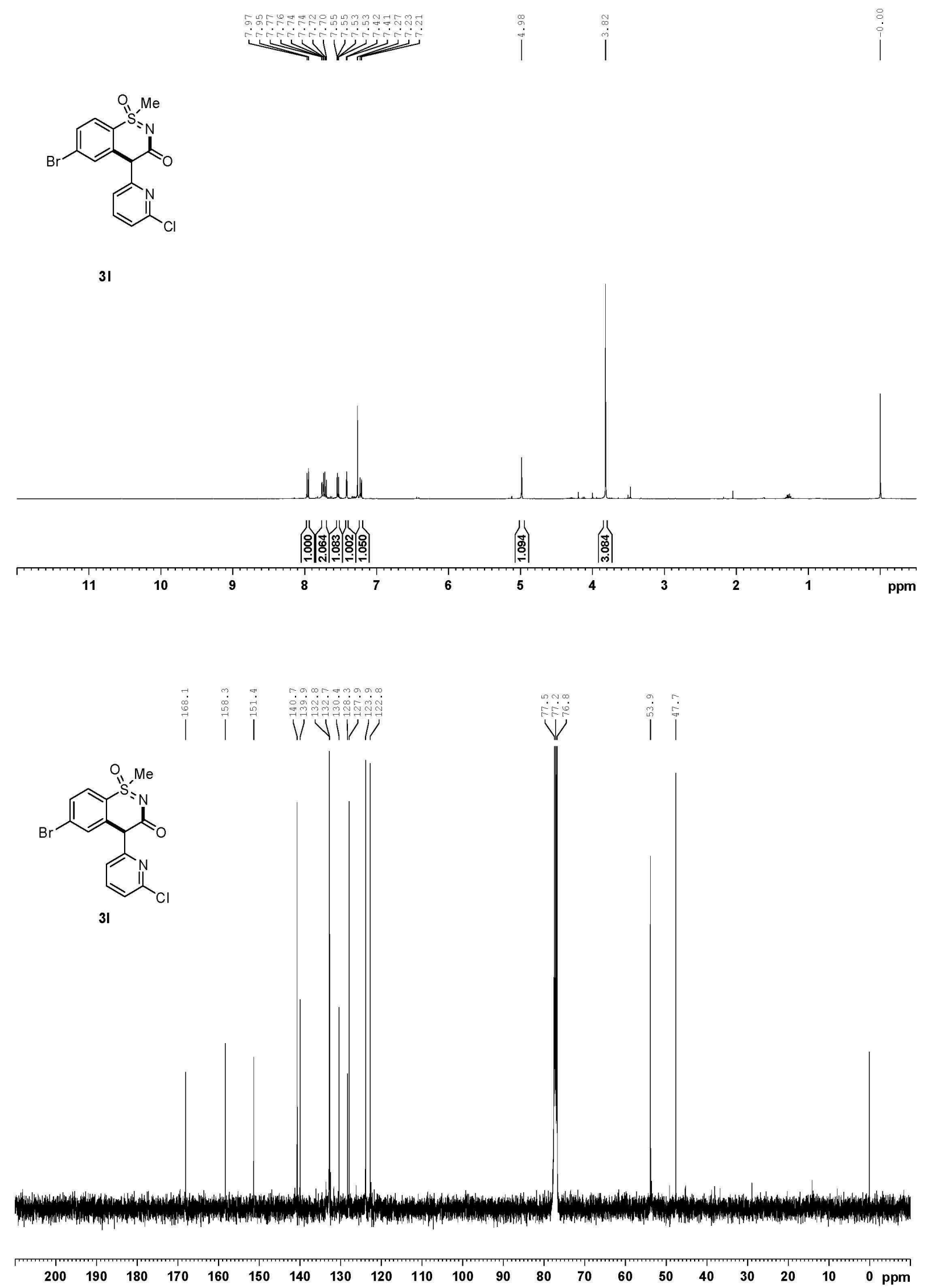


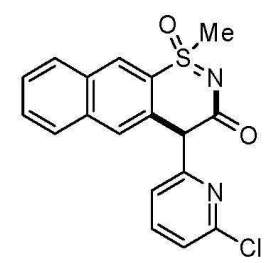

$3 \mathrm{~m}$
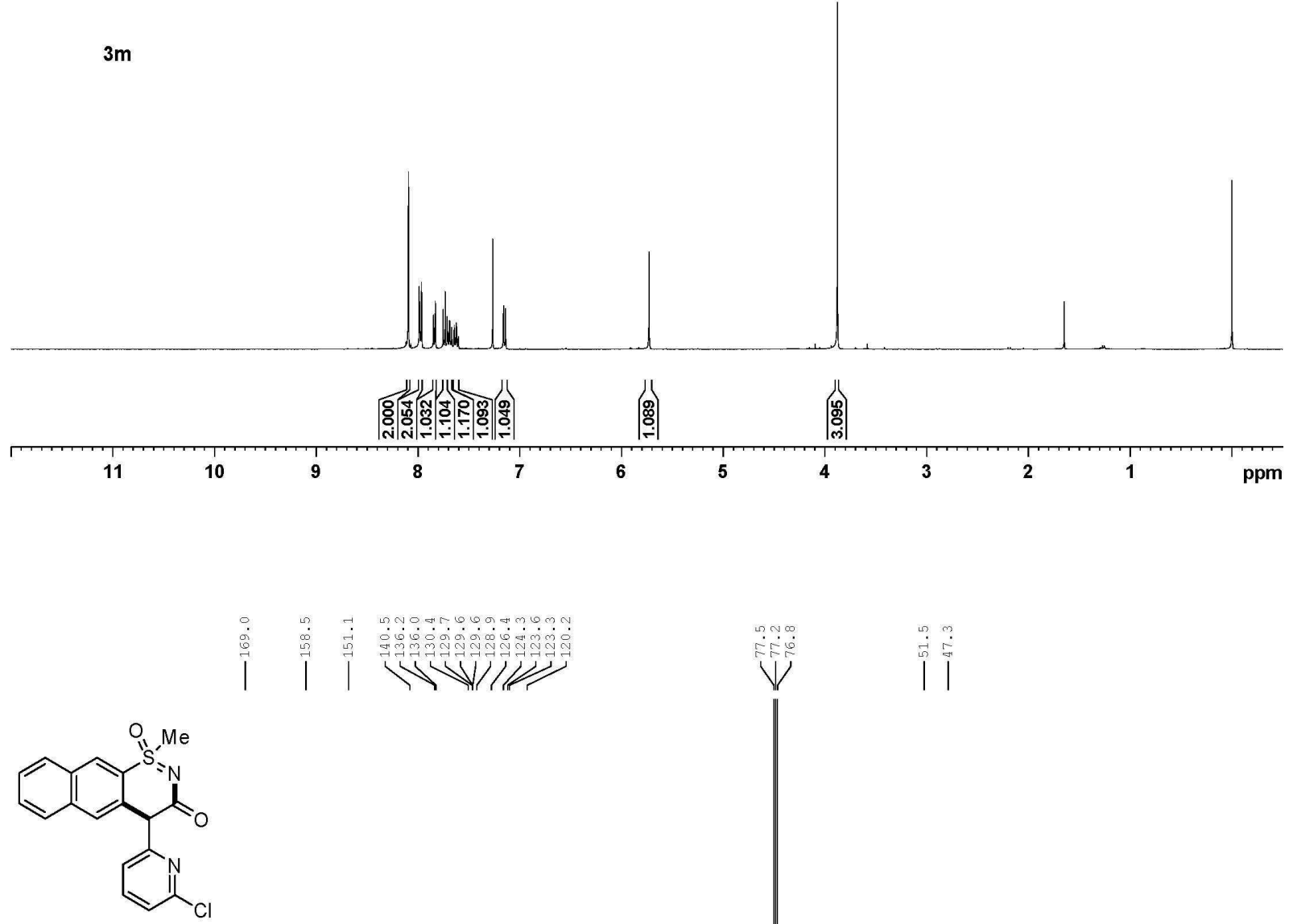

$3 m$

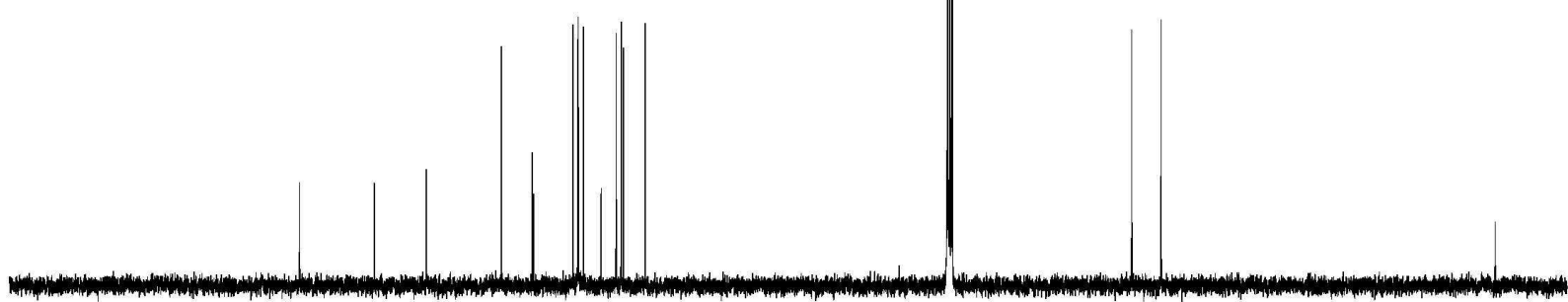

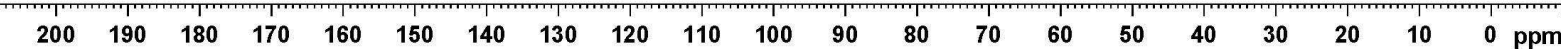



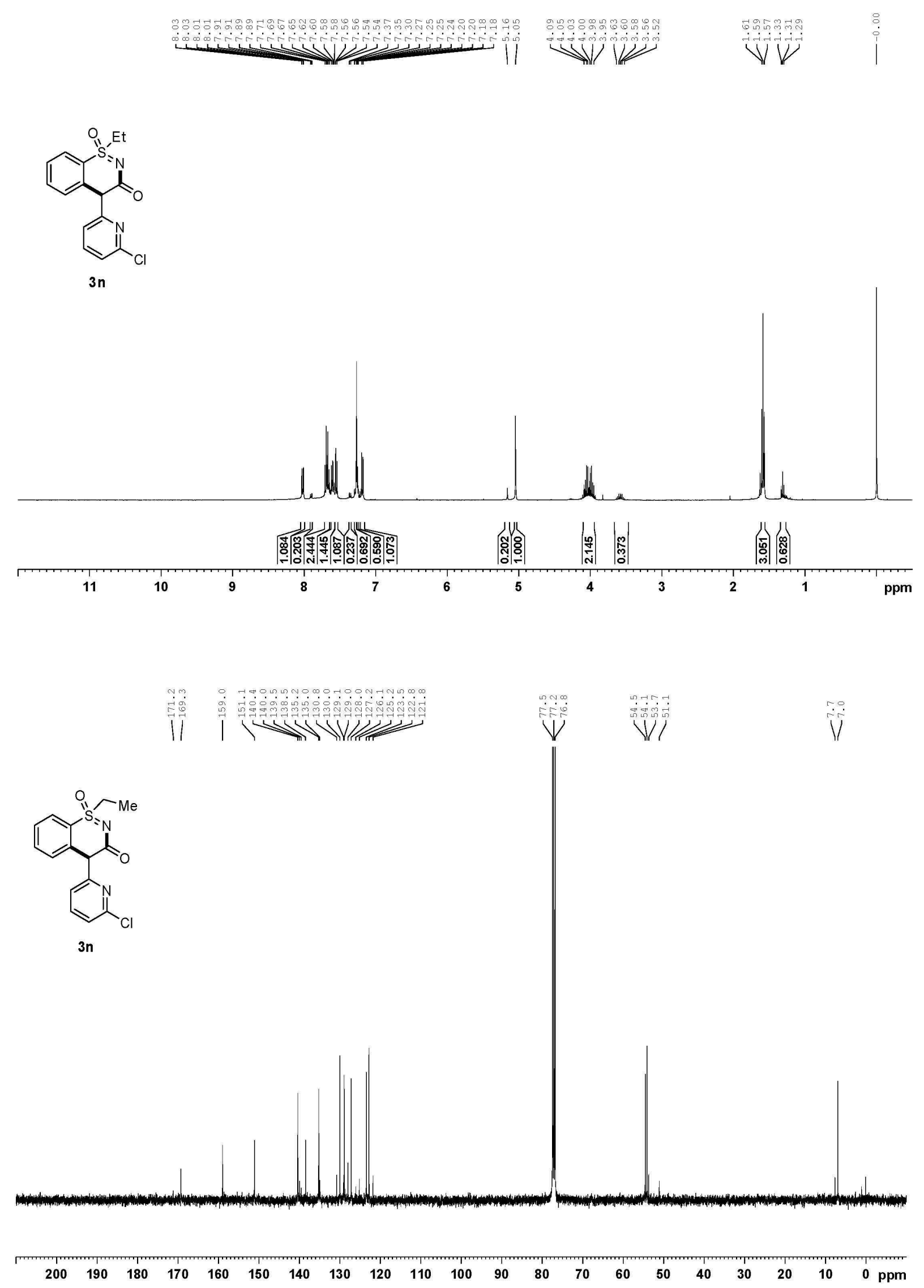
<smiles>CCCP1(=O)N=C(C(N)=O)C(c2cccc(Cl)n2)c2ccccc21</smiles>

30
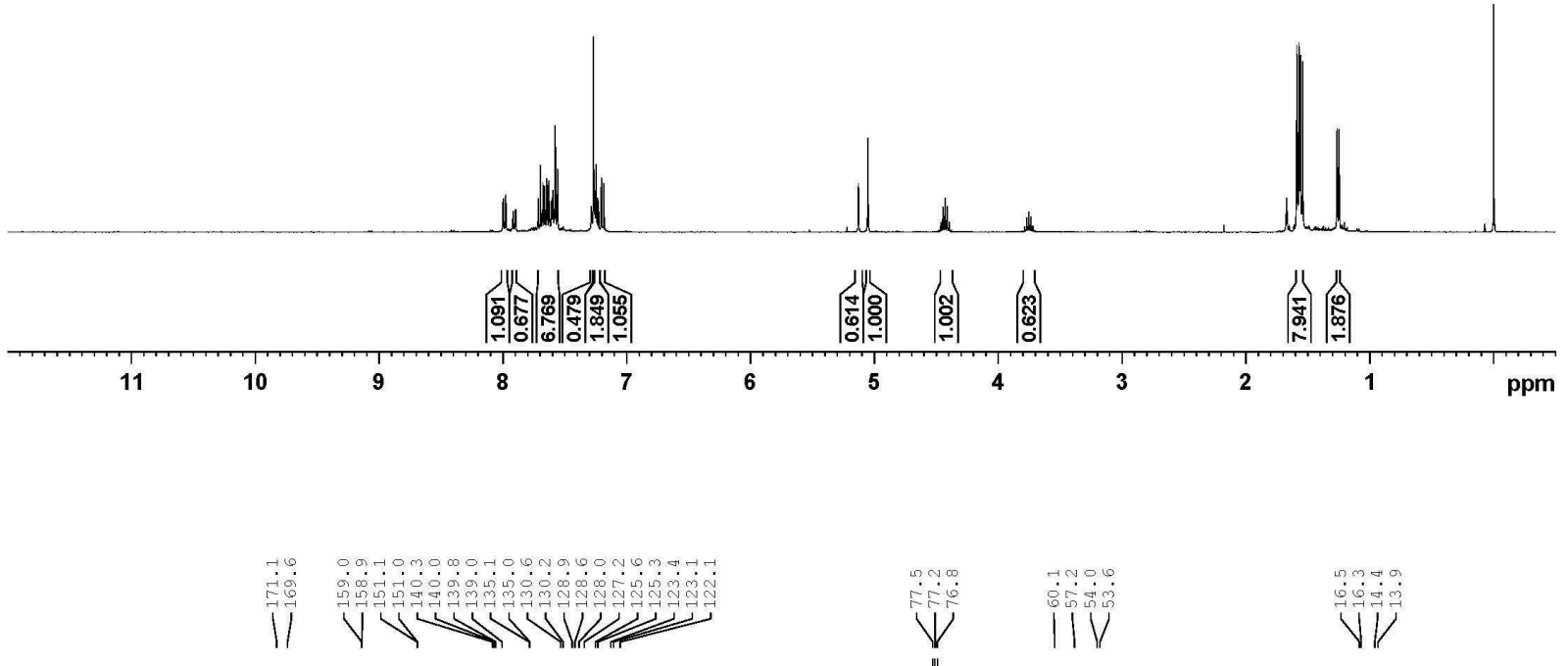<smiles>CC(C)N1C(=O)C(c2cccc(Cl)n2)c2ccccc2S1(=O)=O</smiles>

30

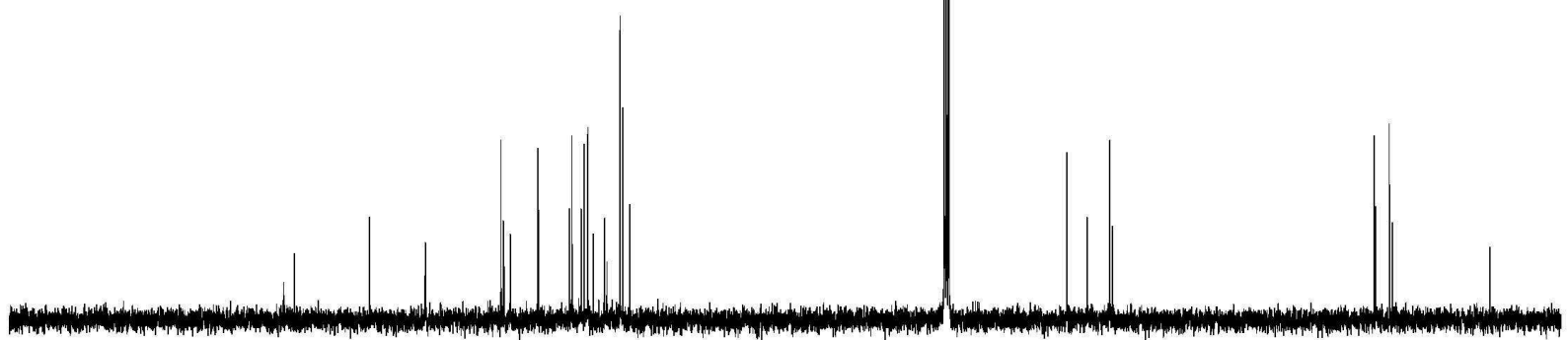

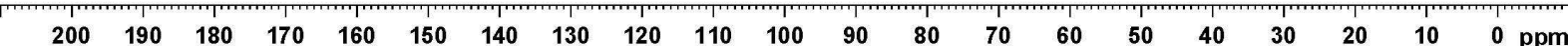



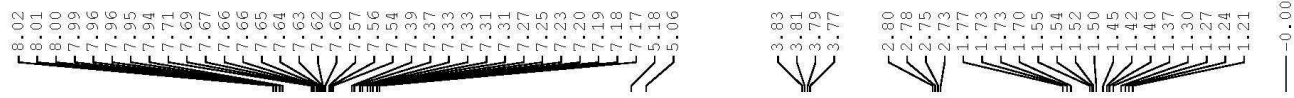<smiles>O=C1N=S(=O)(C2CC2)c2ccccc2C1c1cccc(Cl)n1</smiles>

$3 p$
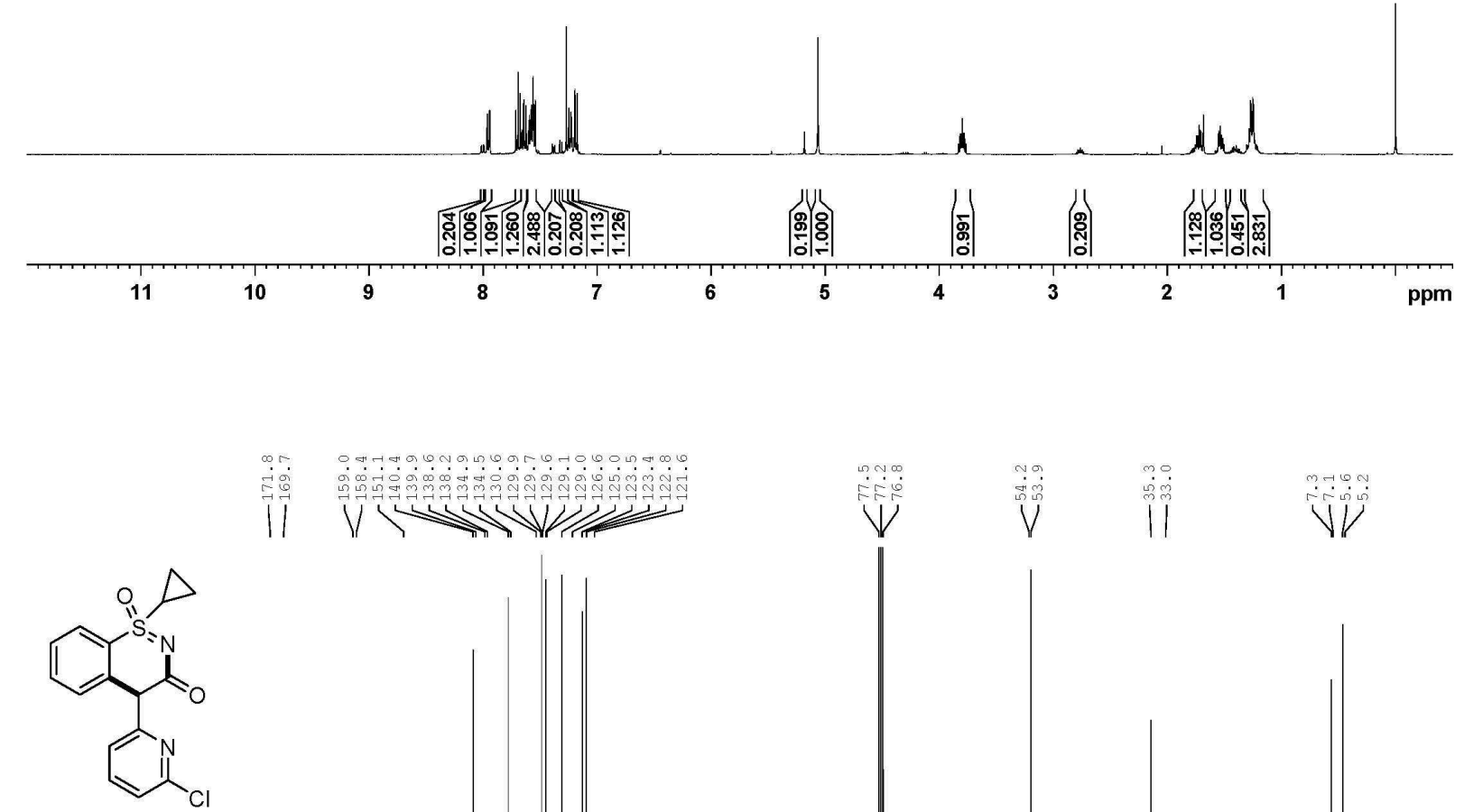

$3 p$
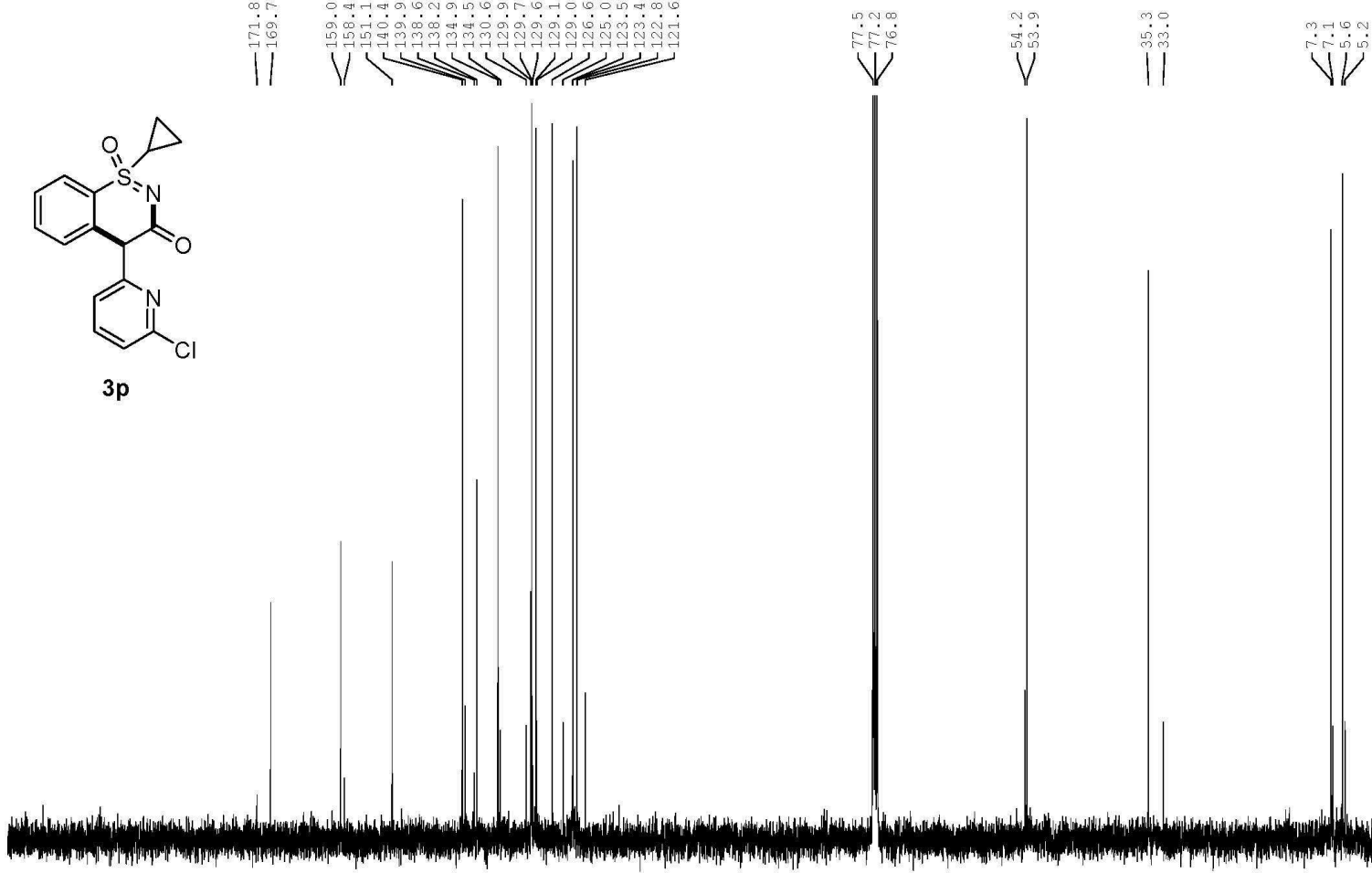

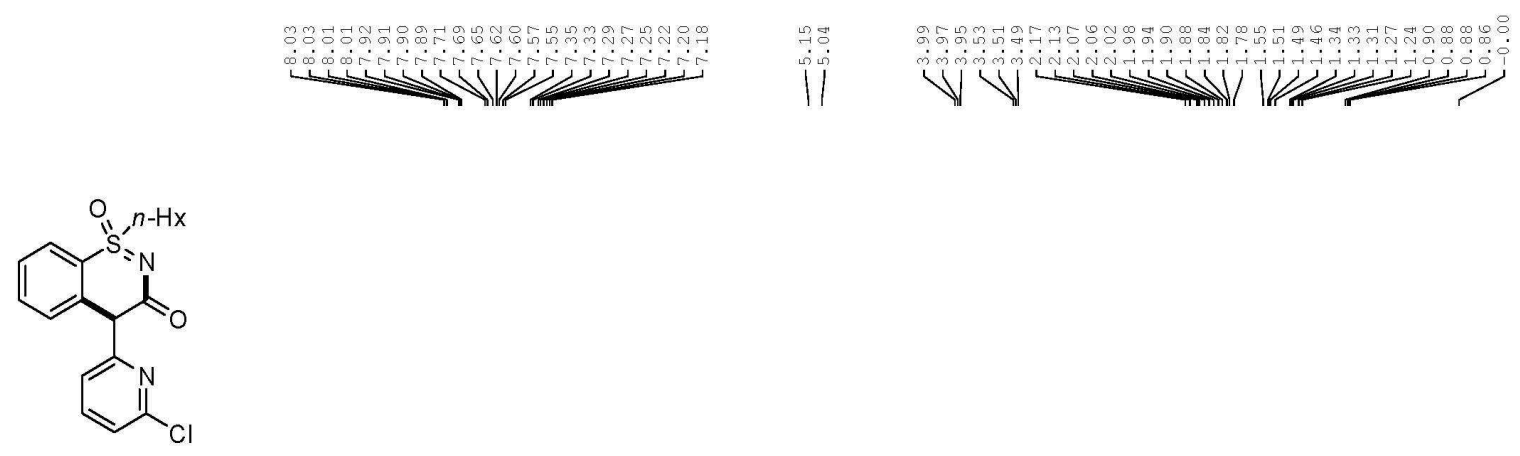

$3 q$
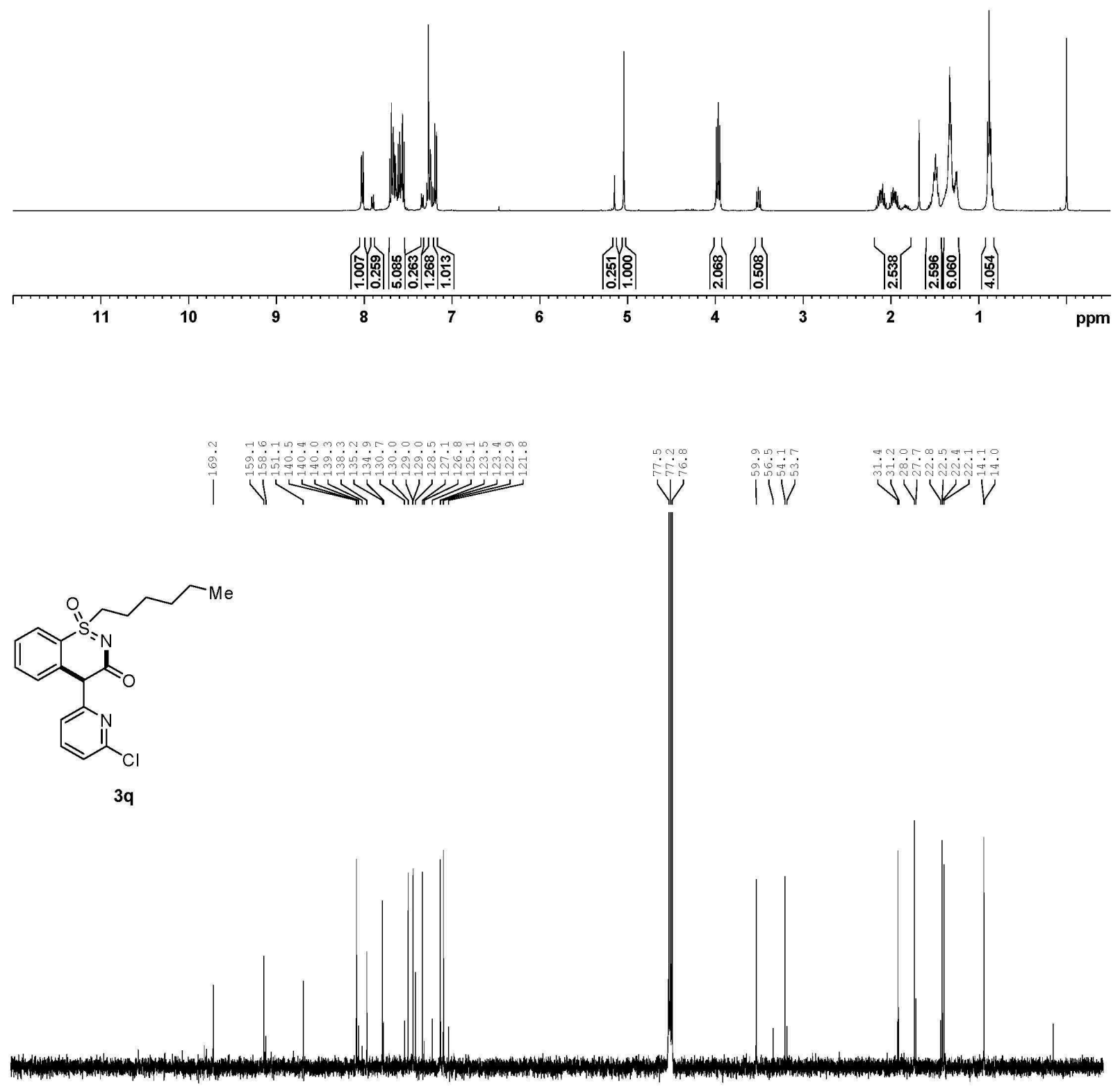

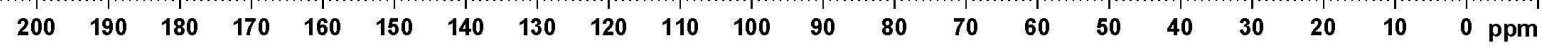




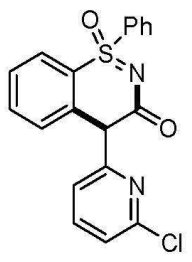

$3 \mathbf{r}$
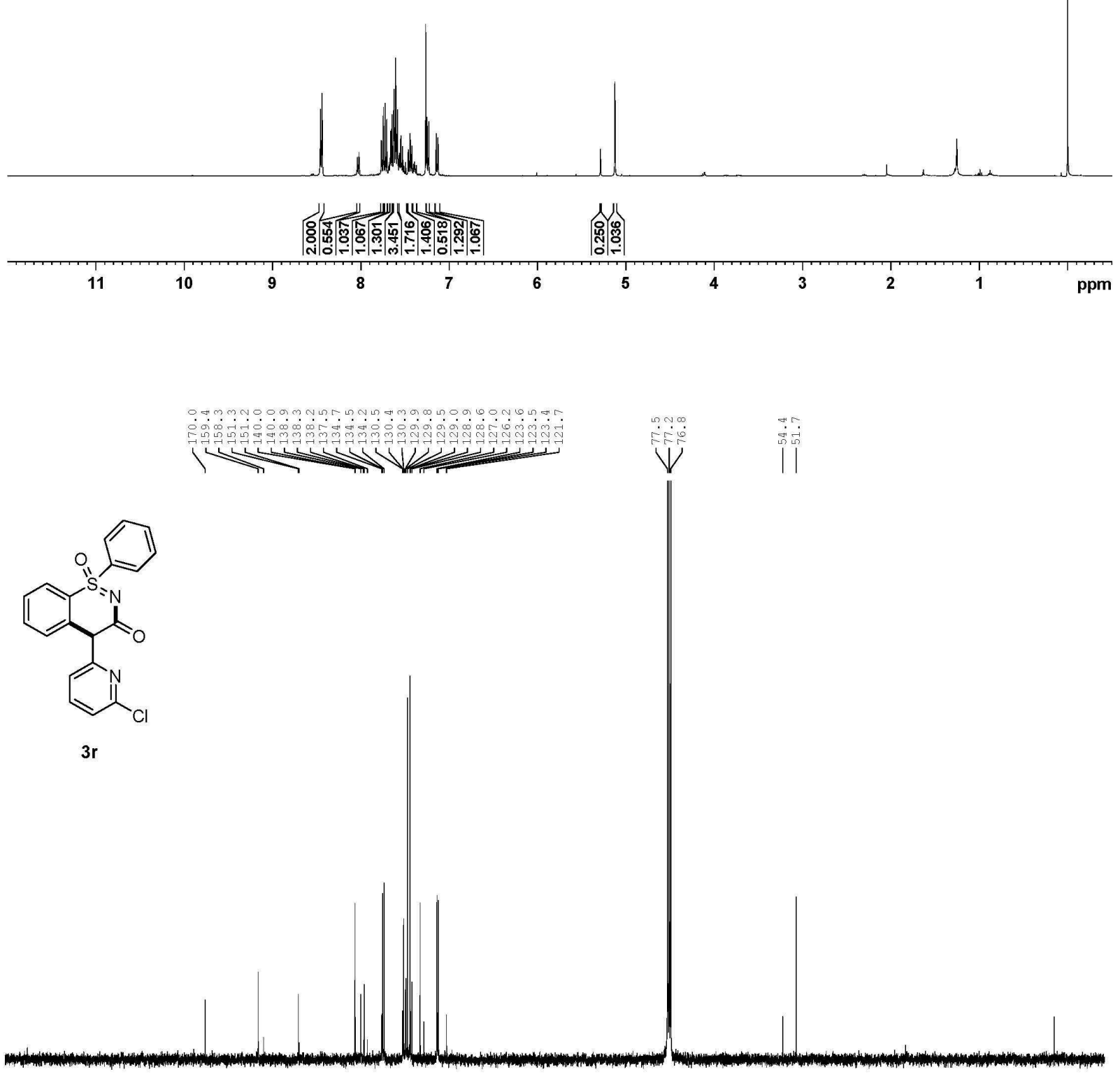

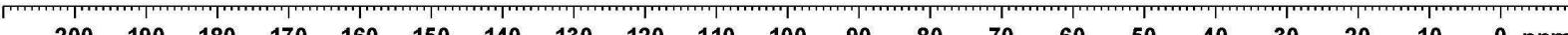
$\begin{array}{llllllllllllllllllllll}200 & 190 & 180 & 170 & 160 & 150 & 140 & 130 & 120 & 110 & 100 & 90 & 80 & 70 & 60 & 50 & 40 & 30 & 20 & 10 & 0 & \mathrm{ppm}\end{array}$ 


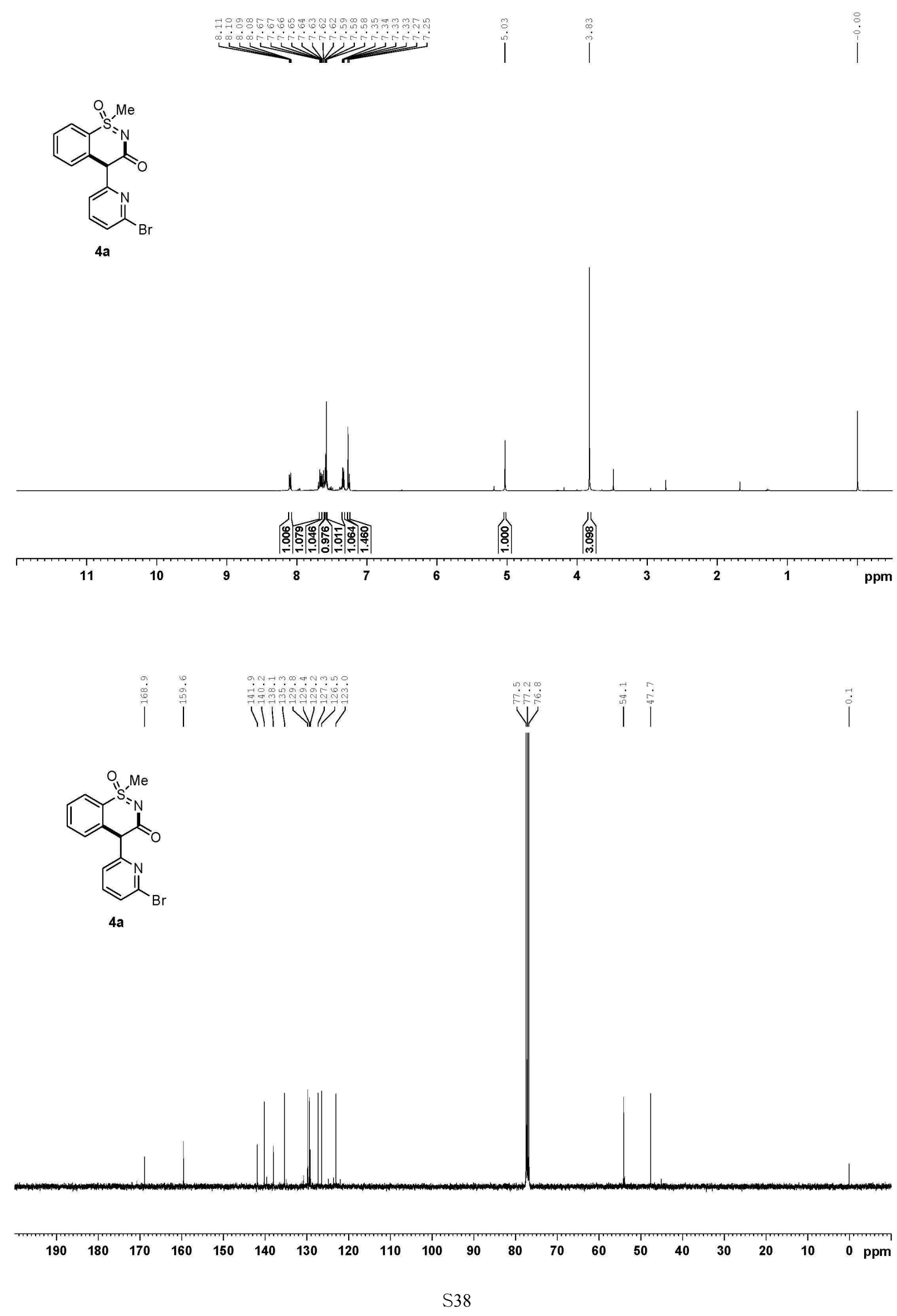



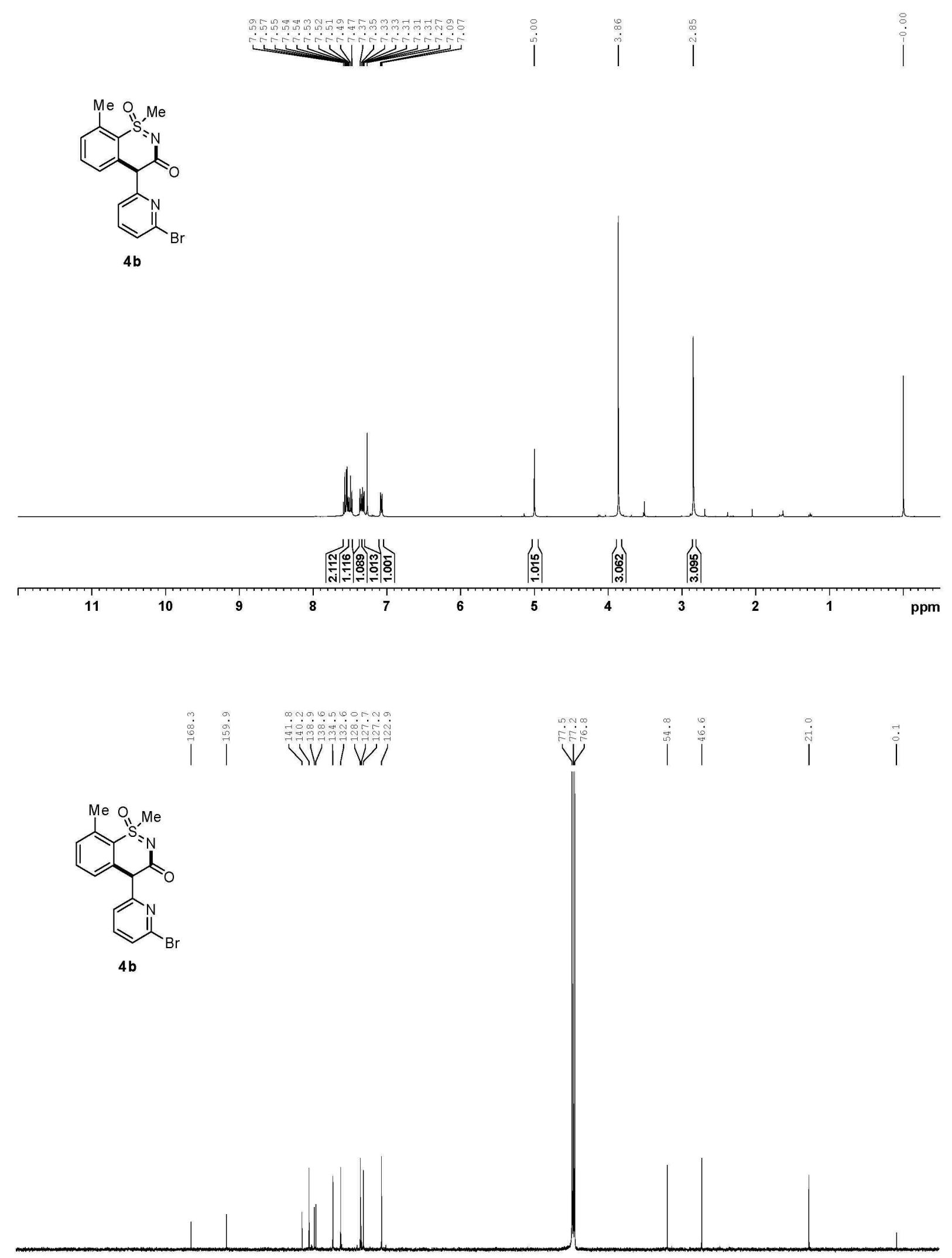

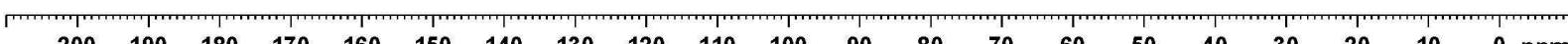
$\begin{array}{lllllllllllllllllllllll}200 & 190 & 180 & 170 & 160 & 150 & 140 & 130 & 120 & 110 & 100 & 90 & 80 & 70 & 60 & 50 & 40 & 30 & 20 & 10 & 0 & \text { ppm }\end{array}$ 

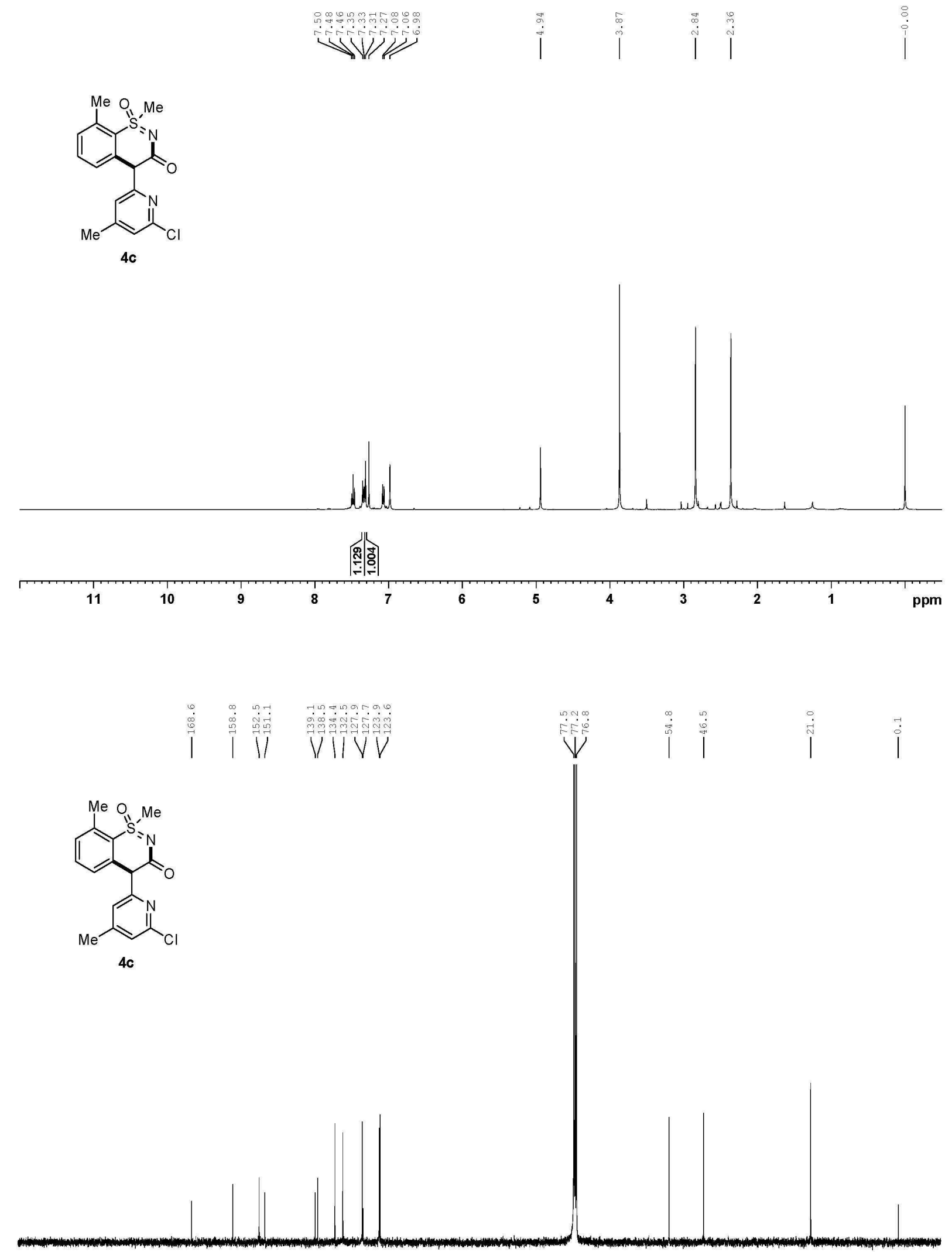

$\begin{array}{llllllllllllllllllllllll}200 & 190 & 180 & 170 & 160 & 150 & 140 & 130 & 120 & 110 & 100 & 90 & 80 & 70 & 60 & 50 & 40 & 30 & 20 & 10 & 0 & \mathrm{ppm}\end{array}$ 


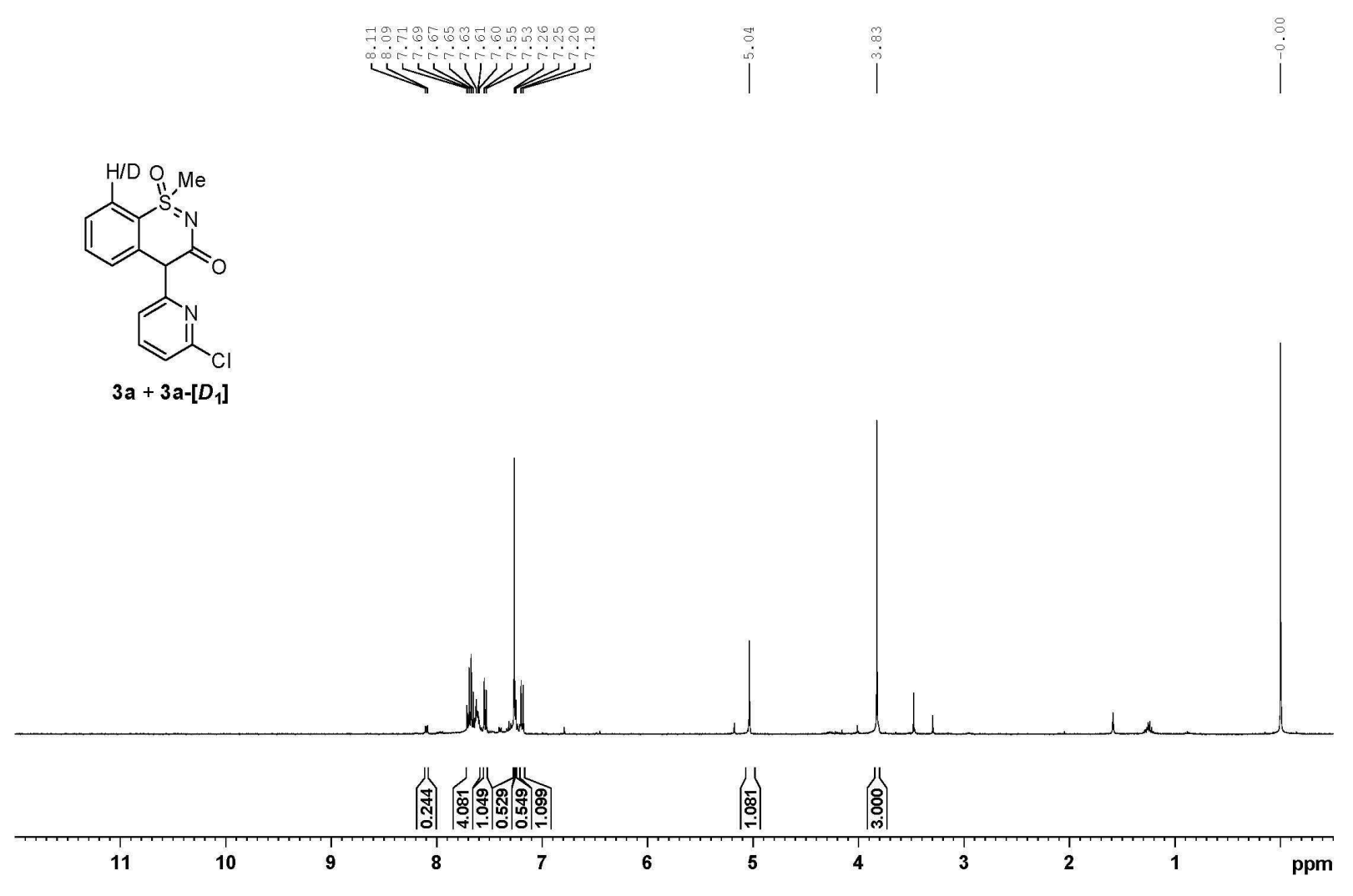

